\title{
FORMES DIFFÉRENTIELLES MÉROMORPHES LOCALEMENT EXACTES
}

\author{
PAR \\ PIERRE DOLBEAULT
}

Introduction. On appellera forme méromorphe localement exacte sur une variété analytique complexe $V$, une forme différentielle méromorphe sur $V$, égale, en chaque point, à la différentielle d'un germe de forme méromorphe. Etant donné un ensemble analytique principal $S$ de $V$, sans singularité, considérons l'espace vectoriel $\varepsilon_{S}^{p+1,0}$ des classes de formes méromorphes localement exactes de degré $p+1$, admettant $S$ comme ensemble polaire à la multiplicité deux au plus, modulo les différentielles des formes méromorphes sur $V$, de degré $p$, admettant $S$ comme ensemble polaire à la multiplicité un au plus.

Dans cet article, nous cherchons à exprimer la dimension (supposée finie) de $\varepsilon_{S}^{p+1,0}$, en fonction des dimensions des espaces de cohomologie des formes différentielles $C^{\infty}$ sur $V$ et sur $S$.

Le §I est consacré à l'étude des faisceaux des germes de résidus relatifs, d'une part, aux faisceaux des germes de $p$-formes méromorphes (resp. méromorphes fermées) admettant $S$ comme ensemble polaire à la multiplicité un au plus, soit $M_{S}^{p}$ (resp. $m_{S}^{p}$ ), d'autre part, au faisceau $d M_{S}^{p}$ (nn. 1 à 3), puis à la recherche de la dimension des espaces de cohomologie de $V$ à coefficients dans les faisceaux des germes de résidus. Pour faciliter l'exposé, nous avons rappelé, au début du $\mathrm{n}^{0} 1$ et au $\mathrm{n}^{0} 2$ des résultats de Kodaira et de Spencer $[4 ; 6]$.

Dans le §II, nous reprenons, à l'aide d'une méthode de D. C. Spencer [6] et en utilisant les résultats du §I, le calcul des dimensions des espaces de cohomologie de $V$ à coefficients dans $M_{S}^{p}, m_{S}^{p}, d M_{S}^{p}$ (Théorème 17); il en résulte les dimensions de $\mathcal{E}_{S}^{p+1,0}$ et d'espaces $\mathcal{E}_{S}^{p+1, q}$ généralisant les espaces de classes de formes méromorphes localement exactes (Théorème 18). Notons que, pour $p>0, d M_{S}^{p}$ est identique au faisceau des germes de $(p+1)$-formes méromorphes fermées admettant $S$ comme ensemble polaire à la multiplicité deux au plus (d'après [2, Corollaire 3.3]).

Dans les deux paragraphes, les calculs n'ont pu être tous menés à bout que dans le cas où $V$ est kählérienne compacte, où l'espace fibré défini par $S$ est suffisamment ample au sens de Kodaira ([3] et [4]) et où $p$ satisfait à certaines inégalités; les calculs reposent alors, de façon essentielle sur le Lemme 6.

Les notations dont la définition n'a pas été rappelée sont celles de [2].

Received by the editors February 16, 1956. 


\section{GERMES DE RESIDUS}

1. Résidus de formes méromorphes. Soient $V$ une variété analytique complexe, de dimension complexe $m$, et $S$ un ensemble analytique principal de $V$, sans singularité. Soit $M_{S}^{p}$ le faisceau des germes de $p$-formes méromorphes dont les germes de diviseurs polaires sont constitués par les germes analytiques définis par $S$, à la multiplicité un au plus. Considérons un recouvrement ouvert $\left\{U_{j}\right\}$ de $V$ assez fin pour qu'il existe des fonctions holomorphes $s_{j}$ telles que $S \cap U_{j}=\left\{s_{j}=0\right\}$; alors, il existe des fonctions holomorphes non nulles $f_{j k}=s_{j} / s_{k}$ sur $U_{j} \cap U_{k} \neq \varnothing$.

Supposons $\left\{U_{j}\right\}$ assez fin pour que l'on puisse choisir, sur chaque $U_{j}$, un système de coordonnées locales $\left(z_{j}^{1}, \cdots, z_{j}^{m}\right)$ tel que $s_{j}(z)=z_{j}^{1}$. On pose $z_{j}^{1}=w_{j} ;\left(z_{j}^{2}, \cdots, z_{j}^{m}\right)=y_{j}$; en particulier, $y_{j}$ est un système de coordonnées locales sur $U_{j} \cap S$. Alors, si $\tau \in\left(M_{S}^{p}\right)_{x}, x \in U_{j}$, il existe un germe de forme holomorphe $\xi_{j}$ tel que $\tau=\xi_{j} / w_{j}$.

Soit $\tau^{\prime}$ une forme méromorphe au voisinage de $x$ définissant le germe $\tau$ en $x$; pour toute forme différentielle $\phi, C^{\infty}$, à support compact dans un voisinage assez petit de $x$, la forme $\tau^{\prime} \wedge \phi$ est localement sommable et définit un courant $\left\langle\tau^{\prime}\right\rangle$ par:

$$
\left\langle\tau^{\prime}\right\rangle[\phi]=\int \tau^{\prime} \wedge \phi .
$$

On désignera par $\langle\tau\rangle$ le germe de courant défini par $\left\langle\tau^{\prime}\right\rangle$ en $x$; ce germe ne dépend pas de la fonction $\tau^{\prime}$ choisie pour définir $\tau$ et on posera conventionnellement

$$
\langle\tau\rangle[\phi]=\int \tau \wedge \phi .
$$

Le faisceau $M_{S}^{p}$ s'identifie au faisceau des germes de courants $\langle\tau\rangle$.

Définition 1. Etant donné $\tau \in\left(M_{S}^{p}\right)_{x}$, si on désigne par $\langle\tau\rangle$ le germe de courant défini par $\tau$, on appelle résidu de $\tau$ le germe de courant $d^{\prime \prime}\langle\tau\rangle$.

L'ensemble des résidus de $M_{S}^{p}$ constitue un faisceau qu'on notera $\Re_{S}^{p}$. Désignons par $\Omega^{p}$ le faisceau des germes de $p$-formes holomorphes sur $V$, la suite

$$
0 \rightarrow \Omega^{p} \rightarrow M_{S}^{p} \stackrel{d^{\prime \prime}}{\rightarrow} Q_{S}^{p} \rightarrow 0,
$$

dans laquelle la seconde flèche désigne l'injection, est évidemment exacte. Dans ce numéro, on se propose d'étudier le faisceau $\mathcal{R}_{S}^{p}$. Pour cela, nous allons d'abord rappeler en détail, des résultats dus à K. Kodaira et à D. C. Spencer [4] concernant le faisceau $M_{S}^{p}$.

Soit $\tau=\xi_{j} / w_{j}$ un élément de $\left(M_{S}^{p}\right)_{x}$, il existe deux germes de formes holomorphes $\xi_{j}^{\prime}$ et $\xi_{j}^{\prime \prime}$ indépendants de $d w_{j}$ tels que:

$$
\xi_{i}=d w_{j} \wedge \xi_{i}^{\prime \prime}\left(w_{i}, y_{j}\right)+\xi_{j}^{\prime}\left(w_{j}, y_{j}\right) ;
$$


en particulier:

$$
\xi_{j}^{\prime}\left(w_{j}, y_{j}\right)=\sum_{\alpha_{1}<\cdots<\alpha_{p}} \xi_{i \alpha_{1} \cdots \alpha_{p}} d y_{j}^{\alpha_{1}} \wedge \cdots \wedge d y_{j}^{\alpha_{p}} .
$$

Sur $U_{j} \cap U_{k} \neq \varnothing$, on a: $w_{j}=f_{j k} w_{k}$ où $f_{j k}$ est holomorphe sans zéro; alors:

$$
\begin{gathered}
d w_{j}=f_{j k} d w_{k}+w_{k} d f_{j k} . \\
\tau=f_{k j} w_{k}^{-1}\left(f_{j k} d w_{k}+w_{k} d f_{j k}\right) \wedge \xi_{j}{ }^{\prime}\left(w_{j}\left(w_{k}, y_{k}\right), y_{j}\left(w_{k}, y_{k}\right)\right) \\
\quad+f_{k j} w_{k}^{-1} \xi_{j}^{\prime}\left(w_{j}\left(w_{k}, y_{k}\right), y_{j}\left(w_{k}, y_{k}\right)\right) ;
\end{gathered}
$$

$\xi_{j}^{\prime}\left(w_{j}\left(w_{k}, y_{k}\right), y_{j}\left(w_{k}, y_{k}\right)\right)$

$$
\begin{gathered}
=d w_{k} \wedge \sum_{\alpha_{1} \cdots \alpha_{p}, \beta_{2}<\cdots<\beta_{p}} \xi_{j \alpha_{1} \cdots \alpha_{p}}\left(\partial y_{j}^{\alpha_{1}} / \partial w_{k}\right)\left(\partial y_{j}^{\alpha_{2}} / \partial y_{k}^{\beta_{2}}\right) \\
\cdots\left(\partial y_{j}^{\alpha_{p}} / \partial y_{k}^{\beta_{p}}\right) d y_{k}^{\beta_{2}} \wedge \cdots \wedge d y_{k}^{\beta_{p}} \\
+\sum_{\alpha_{1} \cdots \alpha_{p}, \beta_{1}<\cdots<\beta_{p}} \xi_{j \alpha_{1} \cdots \alpha_{p}}\left(\partial y_{j}^{\alpha_{1}} / \partial y_{k}^{\beta_{1}}\right) \cdots\left(\partial y_{j}^{\alpha_{p}} / \partial y_{k}^{\beta_{p}}\right) d y_{k}^{\beta_{1}} \wedge \cdots \wedge d y_{k}^{\beta_{p}},
\end{gathered}
$$

le coefficient $\xi_{j \alpha_{1} \cdots \alpha_{p}}$ étant supposé antisymétrique en les $\alpha_{1}, \cdots, \alpha_{p}$. Posons $\xi_{j \alpha}=\sum_{\alpha_{2}<\cdots<\alpha_{p} \neq \alpha} \xi_{j \alpha \alpha_{2}} \cdots \alpha_{p} d y_{j}^{\alpha_{2}} \wedge \cdots \wedge d y_{j}^{\alpha}$. Alors:

$$
\begin{aligned}
& \tau=w_{k}^{-1} d w_{k} \wedge\left[\xi_{j}^{\prime \prime}\left(w_{j}\left(w_{k}, y_{k}\right), y_{j}\left(w_{k}, y_{k}\right)\right)+f_{k j} \sum_{\alpha} \xi_{j \alpha}\left(\partial y_{j}^{\alpha} / \partial w_{k}\right)\right] \\
&+w_{k}^{-1} {\left[w_{k} f_{k j} d f_{j k} \wedge \xi_{j}^{\prime \prime}\left(w_{j}\left(w_{k}, y_{k}\right), y_{j}\left(w_{k}, y_{k}\right)\right)\right.} \\
&\left.+f_{k j} \sum_{\alpha_{1} \cdots \alpha_{p}, \beta_{1}<\cdots<\beta_{p}} \xi_{j \alpha_{1} \cdots \alpha_{p}}\left(\partial y_{j}^{\alpha_{1}} / \partial y_{k}^{\beta_{1}}\right) \cdots\left(\partial y_{j}^{\alpha_{p}} / \partial y_{k}^{\beta_{p}}\right) d y_{k}^{\beta_{1}} \wedge \cdots \wedge d y_{k}^{\beta_{p}}\right] .
\end{aligned}
$$

Désignons par $(\phi)_{S}$ la restriction à $S$ de tout germe $\phi$. Posons: $\eta_{j}^{\prime}=\left(\xi_{j}^{\prime}\right)_{S}$; $\eta_{j}^{\prime \prime}=\left(\xi_{j}^{\prime \prime}\right)_{S}$, alors, pour $x \in U_{j} \cap U_{k} \neq \varnothing$, en comparant les expressions de $\tau \in\left(M_{S}^{p}\right)_{x}$ dans les systèmes de coordonnées $\left(z_{j}\right)$ et $\left(z_{k}\right)$, on obtient:

$$
\begin{aligned}
\eta_{k}^{\prime} & =\left(f_{k j}\right)_{s} \eta_{j}^{\prime} ; \\
\eta_{k}^{\prime \prime} & =\eta_{j}^{\prime \prime}+\left(f_{k j} \sum_{\alpha} \xi_{j \alpha}\left(\partial y_{j}^{\alpha} / \partial w_{k}\right)\right)_{S} .
\end{aligned}
$$

Désignons par $\{S\}$ l'espace fibré à fibre vectorielle de dimension un défini par les fonctions holomorphes non nulles $f_{j k}$ sur $U_{j} \cap U_{k} \neq \varnothing$, par $\{S\}_{S}$ l'espace fibré induit par $\{S\}$ sur $S$ et par $\Omega^{p}\left(\{S\}_{S}\right)$ le faisceau des germes de $p$-formes holomorphes sur $S$, à coefficients dans $\{S\}_{s}$. La formule (3) montre que $\eta_{j}^{\prime} \in \Omega^{p}\left(\{S\}_{S}\right)_{x}$. Tout élément $\tau \in M_{S}^{p}$ definit un élément 
$\eta_{j}^{\prime} \in \Omega^{p}\left(\{S\}_{s}\right)$ d'après (3) et, réciproquement, tout élément $\eta_{j}^{\prime} \in \Omega^{p}\left(\{S\}_{s}\right)_{x}$ est définissable par l'élément $\left(1 / w_{j}\right) \xi_{j}^{\prime} \in\left(M_{S}^{p}\right)_{x}$, où $\xi_{j}^{\prime}\left(0, y_{j}\right)=\eta_{j}^{\prime}$; l'application $r^{\prime}: M_{S}^{p} \rightarrow \Omega^{p}\left(\{S\}_{s}\right)$ qui envoie $\tau$ sur $\eta_{j}^{\prime}$ est donc un épimorphisme.

Remarquons que $M_{S}^{p} \approx \Omega^{p}(\{S\})$, faisceau des germes de $p$-formes holomorphes à coefficients dans $\{S\}$, l'isomorphisme étant défini, sur chaque $U_{j}$, par la multiplication par $w_{j}$; le noyau de $r^{\prime}$ peut donc être considéré comme un sous-faisceau de $\Omega^{p}(\{S\})$, on le notera $\Omega^{\prime \prime p}(\{S\})$. D'où la suite exacte

$$
0 \rightarrow \Omega^{\prime \prime p}(\{S\}) \rightarrow M_{S}^{p} \stackrel{r^{\prime}}{\rightarrow} \Omega^{p}\left(\{S\}_{S}\right) \rightarrow 0[4]
$$

Désignons par $\Omega^{p-1}[S]$ le faisceau des germes de $(p-1)$-formes holomorphes $S$. Si $\tau \in \Omega^{\prime \prime} p(\{S\})$, on a $\eta_{j}^{\prime}=0$, i.e. $\xi_{j \alpha_{1} \cdots \alpha_{p}}=0$ sur $S$, donc

$$
\left(\sum_{\alpha} \xi_{j \alpha}\left(\partial y_{j}^{\alpha} / \partial w_{k}\right)\right)_{S}=0
$$

d'où $\eta_{k}^{\prime \prime}=\eta_{j}^{\prime \prime}$ d'après (4), donc $\eta_{j}^{\prime \prime} \in \Omega^{p-1}[S]$.

Réciproquement, un élément quelconque $\eta_{j}^{\prime \prime} \in \Omega^{p-1}[S]$ est définissable par l'élément $\tau=\left(d w_{j} / w_{j}\right) \wedge \xi_{j}^{\prime \prime}\left(w_{j}, y_{j}\right),\left(\xi_{j}^{\prime \prime}\left(0, y_{j}\right)=\eta_{j}^{\prime \prime}\right)$ de $M_{S}^{p}$ dont l'image par $r^{\prime}$ est nulle. L'application $r^{\prime \prime}: \Omega^{\prime \prime} p(\{S\}) \rightarrow \Omega^{p-1}[S]$ qui envoie $\tau\left(\in \Omega^{\prime \prime p}(\{S\})\right)$ sur $\eta_{j}^{\prime \prime}\left(\in \Omega^{p-1}[S]\right)$ est donc un épimorphisme.

Cherchons le noyau de $r^{\prime \prime}$. Pour que $\tau \in M_{S}^{p}$ appartienne à l'image de $\Omega^{\prime \prime p}(\{S\})_{x} \rightarrow\left(M_{S}^{p}\right)_{x}, \quad\left(x \in U_{j}\right)$, il faut et il suffit que $\tau=\left(d w_{j} / w_{j}\right) \wedge \xi_{j}^{\prime \prime}\left(w_{j}, y_{j}\right)$ $+\left(1 / w_{j}\right) \xi_{j}^{\prime}\left(w_{j}, y_{j}\right)$ a vec $\xi_{j}^{\prime}\left(0, y_{j}\right)=0$, i.e. qu'il existe $\zeta_{j}^{\prime}$ holomorphe tel que $\xi_{j}^{\prime}\left(w_{j}, y_{j}\right)=w_{j} \zeta_{j}^{\prime}\left(w_{j}, y_{j}\right)$. En outre, pour que $\eta_{j}^{\prime \prime}=\xi_{j}^{\prime \prime}\left(0, y_{j}\right)=0$, il faut et il suffit qu'il existe $\xi_{j}^{\prime \prime}\left(w_{j}, y_{j}\right)$ holomorphe tel que: $\eta_{j}^{\prime \prime}\left(w_{j}, y_{j}\right)=w_{j} \zeta_{j}^{\prime \prime}\left(w_{j}, y_{j}\right)$, i.e. que $\tau \in \Omega^{p}$. Donc la suite

$$
0 \rightarrow \Omega^{p} \rightarrow \Omega^{\prime \prime p}(\{S\}) \stackrel{r^{\prime \prime}}{\rightarrow} \Omega^{p-1}[S] \rightarrow 0,
$$

dans laquelle la seconde flèche désigne l'injection, est exacte [4].

Considérons maintenant le résidu de $\tau \in\left(M_{S}^{p}\right)_{x}, x \in U_{j}$, avec $\tau=\left(d w_{j} / w_{j}\right)$ $\wedge \xi_{j}^{\prime \prime}+\left(1 / w_{j}\right) \xi_{j}^{\prime}$. On $a:\langle\tau\rangle[\phi]=\int \tau \wedge \phi=\lim _{\delta \rightarrow 0} \int_{\left|w_{j}\right| \geqq \delta} \tau \wedge \phi$.

$$
\begin{aligned}
d^{\prime \prime}\langle\tau\rangle[\psi] & =(-1)^{p+1}\langle\tau\rangle\left[d^{\prime \prime} \psi\right]=(-1)^{p+1} \lim _{\delta \rightarrow 0} \int_{\left|w_{j}\right| \geqq \delta} \tau \wedge d^{\prime \prime} \psi \\
& =-\lim _{\delta \rightarrow 0} \int_{\left|w_{j}\right| \geqq \delta} d(\tau \wedge \psi)=\lim _{\delta \rightarrow 0} \int_{\left|w_{j}\right|=\delta} \tau \wedge \psi \\
& =\lim _{\delta \rightarrow 0} \int_{\left|w_{j}\right|=\delta}\left[\left(d w_{j} / w_{j}\right) \wedge \xi_{j}^{\prime}\left(w_{j}, y_{j}\right) \wedge \psi+\left(1 / w_{j}\right) \xi_{j}^{\prime}\left(w_{j}, y_{j}\right) \wedge \psi\right] .
\end{aligned}
$$

Posons $\psi=d w_{j} \wedge \psi_{j}^{\prime \prime}+\psi_{j}^{\prime}$ où les formes $\psi_{j}^{\prime \prime}$ et $\psi_{j}^{\prime}$ sont indépendantes de $d w_{j}$, alors: 


$$
\begin{aligned}
d^{\prime \prime}\langle\tau\rangle[\psi] & =\lim _{\delta \rightarrow 0} \int_{\left|w_{j}\right|=\delta}\left(d w_{j} / w_{j}\right) \wedge\left(\xi_{j}^{\prime \prime} \wedge \psi_{j}^{\prime}+(-1)^{p} \xi_{j}^{\prime} \wedge \psi_{j}^{\prime \prime}\right) \\
& =2 \pi i \int_{S} \eta_{i}^{\prime \prime} \wedge \psi_{j}^{\prime}+(-1)^{p} 2 \pi i \int_{S} \eta_{j}^{\prime} \wedge \psi_{j}^{\prime \prime} ;
\end{aligned}
$$

mais, sur $S$, on a $: \eta_{j}^{\prime \prime} \wedge \psi_{j}^{\prime}=\eta_{j}^{\prime \prime} \wedge \psi$, d'où:

$$
d^{\prime \prime}\langle\tau\rangle[\psi]=2 \pi i \int_{S} \eta_{i}^{\prime \prime} \wedge \psi+(-1)^{p} 2 \pi i \int_{S} \eta_{j}^{\prime} \wedge \psi_{i}{ }^{\prime} .
$$

La donnée de $\zeta \in\left(\Omega^{p-1}[S]\right)_{x}$ (c'est-à-dire du germe de courant $\langle\zeta\rangle$ tel que $\left.\langle\zeta\rangle[\psi]=2 \pi i \int_{s} \zeta \wedge \psi\right)$ définit un seul $d^{\prime \prime}\langle\tau\rangle$ avec $\tau=\left(d w_{j} / w_{j}\right) \wedge \xi^{\prime \prime}\left(w_{j}, y_{j}\right)$ et $\xi^{\prime \prime}\left(0, y_{j}\right)=\zeta$. Donc l'application $\lambda: \Omega^{p-1}[S] \rightarrow R_{S}^{p}$ qui envoie $\zeta$ sur $d^{\prime \prime}\langle\tau\rangle$ est un homomorphisme; de plus, d'après l'expression ci-dessus de $d^{\prime \prime}\langle\tau\rangle$, son noyau est nul: $\lambda$ est un monomorphisme. Soit $\theta_{S}^{p}$ le conoyau de $\lambda$.

Proposition 1. Le faisceau $\Theta_{S}^{p}$ qui figure dans la suite exacte

$$
0 \rightarrow \Omega^{p-1}[S] \stackrel{\lambda}{\rightarrow} \Omega_{S}^{p} \stackrel{h}{\rightarrow} Q_{S}^{p} \rightarrow 0,
$$

écrite dans les notations définies ci-dessus, est canoniquement isomorphe au faisceau $\Omega^{p}\left(\{S\}_{S}\right)$.

Démonstration. Considérons le diagramme

(6) $0 \rightarrow \Omega^{p} \rightarrow \Omega^{\prime \prime p}(\{S\}) \stackrel{r^{\prime \prime}}{\rightarrow} \Omega^{p-1}[S] \rightarrow 0$

(8)

$$
i \uparrow Q_{1} \downarrow \quad Q_{2} \quad \lambda \downarrow
$$

(2) $0 \rightarrow \Omega^{p} \rightarrow M_{S}^{p} \quad \stackrel{d^{\prime \prime}}{\rightarrow} \Re_{S}^{p} \quad \rightarrow 0$

$$
\begin{array}{cc}
r^{\prime} \downarrow & h \downarrow \\
\Omega^{p}\left(\{S\}_{s}\right) & Q_{S}^{p} \\
\downarrow & \downarrow \\
0 & 0,
\end{array}
$$

dans lequel les suites sont les suites exactes définies plus haut et $i$ désigne l'identité.

Le carré $Q_{1}$ est commutatif d'après la définition de la suite exacte (6). Le carré $Q_{2}$ est commutatif: en effet, soit $\omega \in \Omega^{\prime \prime} p(\{S\})_{x}$; l'image de $\omega$ dans $M_{S}^{p}$ est $\tau=\left(d w_{j} / w_{j}\right) \wedge \xi_{j}^{\prime \prime}$ pour $x \in U_{j}$ et le germe de forme $\eta_{j}^{\prime \prime}=\left(\xi_{j}^{\prime \prime}\right)_{S}$ ne dépend 
pas de $j$; on a $d^{\prime \prime}\langle\tau\rangle[\psi]=2 \pi i \int_{S} \eta_{j}^{\prime \prime} \wedge \psi$. De plus $r^{\prime \prime} \omega=\eta_{j}^{\prime \prime}$ dont l'image par $\lambda$ est $d^{\prime \prime}\langle\tau\rangle$, ce qui établit la commutativité de $Q_{2}$.

On vérifie alors, formellement, que $\mu=h \circ d^{\prime \prime} \circ r^{\prime-1}$ est un isomorphisme, ce qui établit la Proposition 1.

2. Résidus de formes méromorphes fermées. Soit $m_{S}^{p}$ le sous-faisceau de $M_{S}^{p}$ constitué par les germes $\tau$ de formes fermées; $m_{S}^{p}$ étant considéré comme un faisceau de germes de courants $\langle\tau\rangle, d^{\prime \prime} m_{S}^{p}$ est un sous-faisceau $r_{S}^{p}$ de $R_{S}^{p}$ et on a la suite exacte

$$
0 \rightarrow E^{p} \rightarrow \stackrel{p}{\stackrel{p}{d^{\prime \prime}}} \underset{r_{S}^{p}}{\rightarrow} \rightarrow 0 .
$$

Proposition 2. Le faisceau $r_{S}^{p}$ est canoniquement isomorphe au faisceau $E^{p-1}[S]$ des germes de $(p-1)$-formes holomorphes fermées sur $S[6]$.

Démonstration. Soit $\tau \in\left(m_{\mathrm{S}}^{p}\right)_{x}, x \in U_{j}$; dans les notations du $\mathrm{n}^{\circ} \mathbf{1}$ :

$$
\begin{aligned}
\tau= & \left(d w_{j} / w_{j}\right) \wedge \xi_{j}^{\prime \prime}+\left(1 / w_{j}\right) \xi_{j}^{\prime} ; d \tau=-\left(d w_{j} / w_{j}\right) \wedge d \xi_{j}^{\prime \prime}-\left(d w_{j} / w_{j}^{2}\right) \wedge \xi_{j} \\
& +\left(1 / w_{i}\right) d \xi_{j}^{\prime}=0
\end{aligned}
$$

entraine l'existence d'une forme $\alpha_{j}^{\prime}$ holomorphe telle que $\xi_{j}^{\prime}=w_{j} \alpha_{j}^{\prime}$. Donc: $\tau=\left(d w_{j} / w_{j}\right) \wedge \xi_{j}^{\prime \prime}+\alpha_{j}$; alors $d \tau=-\left(d w_{j} / w_{j}\right) \wedge d \xi_{j}^{\prime \prime}+d \alpha_{j}=0$, donc il existe des formes holomorphes $\beta_{j}$ et $\gamma_{j}$ indépendantes de $d w_{j}$ telles que $d \xi_{j}^{\prime \prime}=d w_{j} \wedge \beta_{j}$ $+w_{j} \gamma_{j}$; il en résulte: $d^{\prime \prime}\langle\tau\rangle[\psi]=2 \pi i \int_{s} \xi_{j}^{\prime \prime} \wedge \psi$ avec $\left(d \xi_{j}^{\prime \prime}\right)_{s}=0$, i.e. $d^{\prime \prime}\langle\tau\rangle$ définit un élément $\left(\xi_{j}^{\prime \prime}\right)_{S} \in\left(E^{p-1}[S]\right)_{x}$. Réciproquement, tout $\eta_{j}^{\prime \prime} \in\left(E^{p-1}[S]\right)_{x}$ est défini par un seul coprant $d^{\prime \prime}\langle\tau\rangle$ avec $\tau=\left(d w_{j} / w_{j}\right) \wedge \xi_{j}^{\prime \prime}\left(w_{j}, y_{j}\right)$ et $\xi_{j}^{\prime \prime}\left(0, y_{j}\right)$ $=\eta_{j}^{\prime \prime}$. Donc l'application $\lambda^{\prime \prime}: r_{S}^{p} \rightarrow E^{p-1}[S]$ qui envoie $d^{\prime \prime}\langle\tau\rangle$ sur $\left(\xi_{j}^{\prime \prime}\right)_{S}$ avec $\left(d \xi_{j}^{\prime \prime}\right)_{S}=0$ est un isomorphisme.

3. Résidus de différentielles. Désignons par $M_{S^{2}}^{p}$ le faisceau des germes de $p$-formes méromorphes dont les diviseurs polaires sont constitués par les germes analytiques définis par $S$ à la multiplicité deux au plus. Nous allons définir et étudier les résidus des éléments du sous-faisceau $d M_{S}^{p}$ de $M_{S^{2}}^{p}$. A tout élément $d \tau \in\left(d M_{S}^{p}\right)_{x}, x \in U_{j}$, associons $\langle d \tau\rangle[\zeta]=\lim _{\delta \rightarrow 0} \int_{\left|w_{j}\right| \geqq \delta} d \tau \wedge \phi$. On sait, d'après un théorème de L. Schwartz [5], que $\langle d \tau\rangle$ est un germe de courant ne dépendant pas de la fonction holomorphe $w_{j}$ qui s'annule sur $S$, au voisinage de $x$, en particulier $\langle d \tau\rangle$ ne dépend pas de $j$. L'ensemble des $\langle d \tau\rangle$ constitue un faisceau canoniquement isomorphe à $d M_{S}^{p}$ qu'on notera $\left\langle d M_{S}^{p}\right\rangle$.

DÉfINITION 2. Etant donné $d \tau \in\left(d M_{S}^{p}\right)_{x}$, si on désigne par $\langle d \tau\rangle$ le germe de courant défini par $d \tau$, on appelle résidu $d e d \tau$ le germe de courant $d^{\prime \prime}\langle d \tau\rangle$.

L'ensemble des $d^{\prime \prime}\langle d \tau\rangle$ constitue un faisceau $S_{S}^{p}$ et $d^{\prime \prime}$ définit un épimorphisme $\left\langle d M_{S}^{p}\right\rangle \rightarrow \delta_{S}^{p}$. Alors, la suite

$$
0 \rightarrow E^{p+1} \rightarrow\left\langle d M_{S}^{p}\right\rangle \stackrel{d^{\prime \prime}}{\rightarrow} \delta_{S}^{p} \rightarrow 0,
$$

où la seconde flèche désigne l'injection, est exacte. 
Proposition 3. $\mathcal{S}_{S}^{p}$ étant le faisceau des germes de résidus de $\left\langle d M_{S}^{p}\right\rangle$, il existe deux homomorphismes $\lambda^{\prime}$ et $h^{\prime}$ tels que, pour $p>0$ la suite

$$
0 \rightarrow E^{p}[S] \stackrel{\lambda^{\prime}}{\rightarrow} S_{S}^{p} \stackrel{h^{\prime}}{\rightarrow} Q_{S}^{p} \rightarrow 0
$$

soit exacte. Le faisceau $\Theta_{S}^{p}$ figure dans la suite exacte (7) et est canoniquement isomorphe a $\Omega^{p}\left(\{S\}_{S}\right)$.

Démonstration. (a) La suite

$$
0 \rightarrow r_{S}^{p} \stackrel{j}{\rightarrow} R_{S}^{p} \stackrel{-d^{\prime}}{\longrightarrow} S_{S}^{p} \rightarrow 0,
$$

dans laquelle $j$ désigne l'injection, est exacte. En effet: $j$ est biunivoque et, d'après un théorème de $L$. Schwartz [5], $\left\langle d M_{S}^{p}\right\rangle=d^{\prime} M_{S}^{p}$ (où $M_{S}^{p}$ est considéré comme faisceau de germes de courants), donc $d^{\prime \prime}\left\langle d M_{S}^{p}\right\rangle=-d^{\prime} d^{\prime \prime} M_{S}^{p}$, i.e. $-d^{\prime}$ est un épimorphisme; reste à montrer que l'image de $j$ est le noyau de $-d^{\prime}$.

Soit $d^{\prime \prime}\langle\sigma\rangle \in r_{S}^{p},\left(\sigma \in m_{S}^{p}\right)$, on a: $-d^{\prime} d^{\prime \prime}\langle\sigma\rangle=d^{\prime \prime} d^{\prime}\langle\sigma\rangle$; d'après le théorème cité de L. Schwartz, $d^{\prime}\langle\sigma\rangle$ est égal à $\langle d \sigma\rangle ; d \sigma=0$ entraîne donc $-d^{\prime} j d^{\prime \prime}\langle\sigma\rangle=0$ : l'image de $j$ est contenue dans le noyau de $-d^{\prime}$.

Soit $\tau \in M_{S}^{p}$, si $d^{\prime \prime}\langle\tau\rangle$ a une image nulle dans $\mathcal{S}_{S}^{p}$, on a: $-d^{\prime} d^{\prime \prime}\langle\tau\rangle=d^{\prime \prime} d^{\prime}\langle\tau\rangle$ $=0$. Mais $d^{\prime}\langle\tau\rangle=\langle d \tau\rangle$ et $d^{\prime \prime}\langle d \tau\rangle=0$ entraîne que $d \tau$ est holomorphe, alors, il existe $\tau^{\prime}$ holomorphe tel que $d \tau=d \tau^{\prime}$ ou $d\left(\tau-\tau^{\prime}\right)=0$, donc $\tau-\tau^{\prime} \in m_{S}^{p}$ et $d^{\prime \prime}\langle\tau\rangle \in r_{S}^{p}$ : le noyau de $-d^{\prime}$ est contenu dans l'image de $j$.

(b) Considérons le diagramme commutatif:

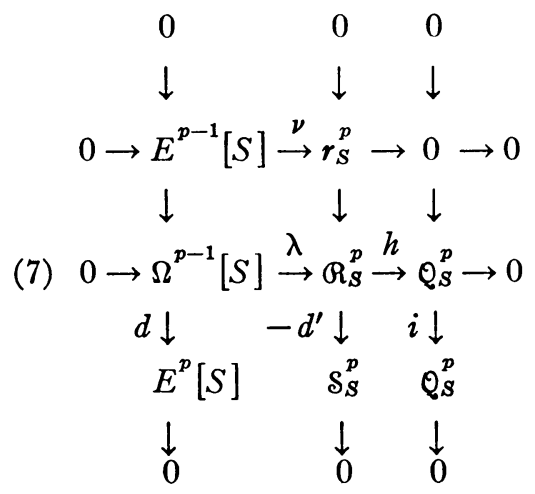

où les suites sont exactes, où $\nu$ est l'isomorphisme de la Proposition 2, et où $i$ désigne l'identité. On vérifie formellement que, en posant $\lambda^{\prime}=-d^{\prime} \circ \lambda \circ d^{-1}$ et $h^{\prime}=-i \circ h \circ d^{\prime-1}$, la suite

$$
0 \rightarrow E^{p}[S] \stackrel{\lambda^{\prime}}{\rightarrow} s_{S}^{p} \stackrel{h^{\prime}}{\rightarrow} Q_{S}^{p} \rightarrow 0
$$


est exacte. Compte tenu de la Proposition 1, on obtient la Proposition 3.

4. Cohomologie à coefficients dans $r_{S}^{p}$ et $\mathcal{R}_{S}^{p}$. On se propose maintenant de déterminer la dimension des espaces de cohomologie à coefficients dans les faisceaux de germes de résidus.

Proposition 4. Si $V$ est une variêté analytique complexe et $S$ un ensemble analytique principal de $V$ sans singularité, on $a$ :

$$
\operatorname{dim} H^{q}\left(V, r_{S}^{p}\right)=\operatorname{dim} K^{p-1, q}(S, C) .
$$

Démonstration. D'après la Proposition 2, on a:

$$
H^{q}\left(V, r_{S}^{p}\right) \approx H^{q}\left(V, E^{p-1}[S]\right) ;
$$

d'après la définition de $E^{p-1}[S]$, le dernier espace est isomorphe à $H^{q}\left(S, E^{p-1}[S]\right)$, ce qui est isomorphe à $K^{p-1, q}(S, C)$ d'après le Théorème 1.6 de [2].

Calculons maintenant $\operatorname{dim} H^{q}\left(V, \Re_{S}^{p}\right)$.

La suite (7) $0 \rightarrow \Omega^{p-1}[S] \rightarrow \mathbb{R}_{S}^{p} \rightarrow Q_{S}^{p} \rightarrow 0$ définit la suite exacte de cohomologie:

$$
\begin{aligned}
0 & \rightarrow \text { conoyau }\left[H^{q-1}\left(V, \mathcal{R}_{S}^{p}\right) \rightarrow H^{q-1}\left(V, \Theta_{S}^{p}\right)\right] \rightarrow H^{q}\left(V, \Omega^{p-1}[S]\right) \rightarrow H^{q}\left(V, \mathcal{R}_{S}^{p}\right) \\
& \rightarrow H^{q}\left(V, \Theta_{S}^{p}\right) \rightarrow \text { conoyau }\left[H^{q}\left(V, \mathcal{Q}_{S}^{p}\right) \rightarrow H^{q}\left(V, \Theta_{S}^{p}\right)\right] \rightarrow 0 .
\end{aligned}
$$

Cette suite permet d'évaluer $\operatorname{dim} H^{q}\left(V, \mathfrak{R}_{S}^{p}\right)$ connaissant dim conoyau $\left[H^{t}\left(V, \Re_{S}^{p}\right) \rightarrow H^{t}\left(V, \Theta_{S}^{p}\right)\right]$ pour $t=q-1$ et $t=q$; c'est ce nombre qu'on va calculer. On a le diagramme:
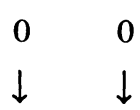

(6) $0 \rightarrow \Omega^{p} \rightarrow \Omega^{\prime \prime p}(\{S\}) \rightarrow \Omega^{p-1}[S] \rightarrow 0$

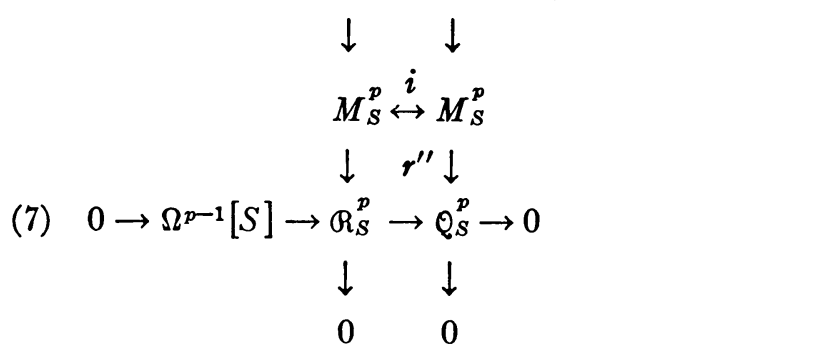

dans lequel les suites sont exactes. 
La suite (5') se déduit de (5) avec $r^{\prime \prime}=\mu \circ r^{\prime}$ où $\mu$ est l'isomorphisme de la Proposition $1 ; i$ désigne l'identité. La commutativité de (8) entraîne celle de (15). Par le raisonnement formel utilisé dans la partie (c) de la démonstration du Théorème 1.10 de [2], on montre que le diagramme (15) définit le diagramme commutatif de cohomologie suivant dans lequel les suites sont exactes et où $i$ désigne l'identité:

$$
\begin{array}{lll}
H^{t}\left(V, Q_{S}^{p}\right) \rightarrow & H^{t}\left(V, Q_{S}^{p}\right) & \rightarrow H^{t+1}\left(V, \Omega^{p-1}[S]\right) \\
\downarrow & \downarrow & i \uparrow \\
H^{t+1}\left(V, \Omega^{p}\right) \rightarrow & H^{t+1}\left(V, \Omega^{\prime p}(\{S\})\right) \rightarrow H^{t+1}\left(V, \Omega^{p-1}[S]\right) \stackrel{k}{\rightarrow} H^{t+2}\left(V, \Omega^{p}\right) .
\end{array}
$$

Alors, on a:

$$
\begin{aligned}
\text { conoyau }\left[H^{t}\left(V, \mathcal{R}_{S}^{p}\right) \rightarrow\right. & H^{t}\left(V, Q_{S}^{p}\right] \approx \text { image }\left[H^{t}\left(V, Q_{S}^{p}\right) \rightarrow H^{t+1}\left(V, \Omega^{p-1}[S]\right)\right] \\
& \subset \text { image }\left[H^{t+1}\left(V, \Omega^{\prime p} p(\{S\})\right) \rightarrow H^{t+1}\left(V, \Omega^{p-1}[S]\right)\right] \\
& \approx \text { noyau }\left[H^{t+1}\left(V, \Omega^{p-1}[S]\right) \stackrel{k}{\rightarrow} H^{t+2}\left(V, \Omega^{p}\right)\right] .
\end{aligned}
$$

On a: $H^{t+1}\left(V, \Omega^{p-1}[S]\right) \approx H^{t+1}\left(S, \Omega^{p-1}[S]\right)$ et, d'après le Théorème $1.4 \mathrm{de}$ [2] des isomorphismes:

$$
\begin{aligned}
& \delta_{1}: H^{t+1}\left(S, \Omega^{p-1}[S]\right) \approx H^{p-1, t+1}(S, C) ; \\
& \delta_{2}: H^{t+2}\left(V, \Omega^{p}\right) \approx H^{p, t+2}(V, C) ;
\end{aligned}
$$

si l'on identifie les deux espaces $H^{t+1}\left(V, \Omega^{p-1}[S]\right)$ et $H^{t+1}\left(S, \Omega^{p-1}[S]\right)$, l'homomorphisme $k$ définit l'homomorphisme

$$
k^{\prime}=\delta_{2}^{-1} \circ k \circ \delta_{1}: H^{p-1, t+1}(S, C) \rightarrow H^{p, t+2}(V, C) .
$$

On va déterminer le noyau de $k^{\prime}$ qui est isomorphe au noyau de $k$.

Lemme 5. Dans les notations ci-dessus, l'homomorphisme $k^{\prime}$ est défini par l'application de la classe de $d^{\prime \prime}$-cohomologie de toute $(p-1, t+1)$-forme $d^{\prime \prime}$-fermée $\omega$ de $S$ sur la classe de $d^{\prime \prime}$-cohomologie du courant $\omega^{\prime}, d^{\prime \prime}$-fermé, de type $(p, t+2)$ de $V$ tel que $\omega^{\prime}[\psi]=2 \pi i \int_{s} \omega \wedge \psi$.

Démonstration. (a) Explicitons l'homomorphisme k. Si $\tau \in\left(\Omega_{j}^{\prime \prime} p(\{S\})\right)_{x}$, on a vu, au no 1 , qu'il existe deux germes de formes holomorphes $\xi_{j}^{\prime \prime}$ et $\zeta_{j}$ indépendants de $d w_{j}$ tels que $\tau=\left(d w_{j} / w_{j}\right) \wedge \xi_{j}^{\prime \prime}\left(w_{j}, y_{j}\right)+\zeta_{j}^{\prime}\left(w_{j}, y_{j}\right)$ et que $\left(\xi_{j}^{\prime \prime}\right)_{S}=\left(\xi_{k}^{\prime \prime}\right)_{s}$ si $x \in U_{j} \cap U_{k} \neq \varnothing$.

Soit $\alpha \in H^{t+1}\left(V, \Omega^{p-1}[S]\right)$. Pour un recouvrement $r=\left\{U_{j}\right\}$ assez fin, de nerf $N_{r}$, la classe $\alpha$ est définie comme l'image canonique de la classe de cohomologie d'un cocycle $\left\{\alpha_{j_{0} \ldots j_{t+1}}\right\}$ de $N_{r}$ à coefficients dans $\Omega^{p-1}[S]$. On a: 


$$
\sum_{l}(-1)^{l} \alpha_{i_{0}} \cdots_{i} \cdots i_{t+2}=0
$$

or

$$
\alpha_{j_{0}} \cdots j_{t+1}
$$

est l'image, dans l'homomorphisme

$$
\Gamma\left(U_{i_{0}} \cap \cdots \cap U_{i_{t+1}}, \Omega^{\prime \prime p}(\{S\})\right) \rightarrow \Gamma\left(U_{i_{0}} \cap \cdots \cap U_{i_{t+1}}, \Omega^{p-1}[S]\right)
$$

d'un courant

$$
\tau_{j_{0} \cdots j_{t+1}}=\left(d w_{j_{0}} / w_{j_{0}}\right) \wedge \xi_{j_{0} \cdots j_{t+1}}^{\prime \prime}\left(w_{j}, y_{j}\right)
$$

avec

$$
\left(\xi_{j_{0}}^{\prime \prime} \cdots j_{t+1}\right)_{s}=\alpha_{j_{0}} \cdots i_{t+1}
$$

Alors (17) entraine:

$$
\sum_{l}(-1)^{l} \tau_{i_{0}} \ldots i_{l} \cdots i_{t+2}=\gamma_{i_{0}} \cdots i_{t+2} \in \Gamma\left(U_{i_{0}} \cap \cdots \cap U_{i_{t+2}}, \Omega^{p}\right) .
$$

Donc le cocycle $\left\{\gamma_{i_{0} \ldots i_{t+2}}\right\}$ est cohomologue à $\left\{\gamma_{i_{0}}^{\prime} \ldots i_{t+2}\right\}$ avec:

$$
\gamma_{i_{0} \cdots i_{t+2}}^{\prime}=\left(d w_{i_{0}} / w_{i_{0}}\right) \wedge \sum_{l}(-1)^{l} \xi_{i_{0} \ldots i_{1} \cdots i_{t+2}}^{\prime \prime}
$$

Puisque $\gamma_{i_{0}}^{\prime} \ldots i_{t+2}$ est holomorphe, il existe une forme holomorphe $\zeta_{i_{0}}^{\prime \prime} \cdots i_{i_{+2}}$ sur $U_{i_{0}} \cap \cdots \cap U_{i_{t+2}}$ telle que:

$$
\sum_{l}(-1) l \xi_{i_{0} \cdots i_{t} \cdots i_{t+2}}^{\prime \prime}=w_{i_{0}} \zeta_{i_{0} \cdots i_{t+2}}^{\prime \prime}
$$

Alors:

$$
\gamma_{i_{0} \cdots i_{t+2}}^{\prime}=d w_{i_{0}} \wedge \zeta_{i_{0} \cdots i_{t+2}}^{\prime \prime}
$$

L'image canonique de la classe de cohomologie du cocycle $\left\{\gamma_{i_{0}}^{\prime} \ldots i_{i_{+2}}\right\}$ dans $H^{t+2}\left(V, \Omega^{p}\right)$ est $k \alpha$ par définition de $k$.

(b) Soit $\delta_{2}^{\prime}$ l'isomorphisme: $H^{t+1}\left(V, F^{p, 1}\right) \rightarrow H^{t+2}\left(V, \Omega^{p}\right)$ qui intervient dans la démonstration du Théorème 1.4 de [2]. Pour expliciter l'homomorphisme $k^{\prime}$ on va d'abord expliciter l'homomorphisme $k^{\prime \prime}=\left(\delta_{2}^{\prime}\right)^{-1} \circ k$. Etant donné $\alpha \in H^{t+1}\left(V, \Omega^{p-1}[S]\right)$, déterminons $\left(\delta_{2}^{\prime}\right)^{-1} \circ k \alpha$; d'après (18), (19) et la démonstration du Théorème 1.4 de [2], c'est l'image canonique, dans $H^{t+1}\left(V, F^{p, 1}\right)$, de la classe de cohomologie du cocycle $\left\{d^{\prime \prime}\left\langle\tau_{j_{0} \cdots j_{t+1}}\right\rangle\right\}$. Alors, pour (support v) $\subset U_{j_{0}} \cap \cdots \cap U_{j_{t+1}}$, on a:

$$
d^{\prime \prime}\left\langle\tau_{j_{0} \cdots i_{t+1}}\right\rangle[v]=2 \pi i \int_{S} \xi_{j_{0}}^{\prime \prime} \cdots i_{t+1} \wedge v=2 \pi i \int_{S} \alpha_{j_{0} \cdots i_{t+1}} \wedge v .
$$


L'homomorphisme $\left(\delta_{2}^{\prime}\right)^{-1} \circ k$ applique donc l'image canonique, dans $H^{t+1}\left(V, \Omega^{p-1}[S]\right)$, de la classe de $d^{\prime \prime}$-cohomologie du cocycle $\left\{\alpha_{j_{0} \cdots j_{t+1}}\right\}$ de $N_{r}$ sur l'image canonique, dans $H^{t+1}\left(V, F^{p, 1}\right)$, de la classe de cohomologie du cocycle $\left\{d^{\prime \prime}\left\langle\tau_{j_{0} \cdots j_{t+1}}\right\rangle\right\}$ de $N_{r}$ tel que:

$$
d^{\prime \prime}\left\langle\tau_{j_{0} \cdots i_{t+1}}\right\rangle[v]=2 \pi i \int_{S} \alpha_{j_{0} \cdots i_{t+1}} \wedge v
$$

On sait qu'il existe un isomorphisme canonique

$$
\delta_{2}^{\prime \prime}: H^{p, t+2}(V, C) \rightarrow H^{t+1}\left(V, F^{p, 1}\right)
$$

tel que $\delta_{2}=\delta_{2}^{\prime} \circ \delta_{2}^{\prime \prime}$. Alors, il est clair, d'après la démonstration du Théorème 1.4 de [2] que l'homomorphisme $k^{\prime}=\left(\delta_{2}^{\prime \prime}\right)^{-1} \circ k^{\prime \prime} \circ \delta_{1}$ est défini comme il est dit dans le Lemme 5.

Lemme 6. Si $V$ est une variêté kählérienne compacte, si $S$ est un ensemble analytique principal sans singularité de $V$, et si $\{S\}$ est suffisamment ample, l'homomorphisme $a: K^{0, q}(S, C) \rightarrow K^{0, q+2}(V, C)$ qui associe, a la classe de cohomologie de toute $q$-forme fermée $\omega$ sur $S$, la classe de cohomologie du courant $\omega^{\prime}$ sur $V$ défini par $\omega^{\prime}[\psi]=\int_{S} \omega \wedge \psi$ possède les propriétés suivantes:

si $q<m-3$ ou $q \geqq m$, c'est un monomorphisme;

si $q=m-1$, c'est un épimorphisme.

Démonstration. Désignons par $\mathfrak{d}$ et $\mathfrak{d}^{\prime}$ les isomorphismes $H^{q+2}(V, C)$ $\rightarrow H_{2 m-q-2}(V, C)$ et $H^{q}(S, C) \rightarrow H_{2 m-q-2}(S, C)$ du théorème de dualité des variétés, par $\rho$ et $\rho^{\prime}$ les isomorphismes $K^{0, q+2}(V, C) \rightarrow H^{q+2}(V, C)$ et $K^{0, q}(S, C)$ $\rightarrow H^{q}(S, C)$ du théorème de de Rham, et par $j$ l'homomorphisme $H_{2 m-q-2}(S, C)$ $\rightarrow H_{2 m-q-2}(V, C)$ induit par l'inclusion de $S$ dans $V$. D'après la définition de $a$ on a: $a=\rho^{-1} \circ \delta^{-1} \circ j \circ \delta^{\prime} \circ \rho^{\prime}$.

Soient $j^{*}: H^{2 m-q-2}(V, C) \rightarrow H^{2 m-q-2}(S, C)$ et $j_{0}^{*}: H^{q+2}(V, C) \rightarrow H^{q+2}(S, C)$ les homomorphismes induits par l'inclusion de $S$ dans $V$.

(1) Supposons $q+2>m$, on a: $2 m-q-2 \leqq m-1$; alors, si $V$ est kählérienne compacte et si $\{S\}$ est suffisamment ample au sens de Kodaira $[3 ; 4]$, d'après le Théorème 2 de [4], on a:

(a) $q \geqq m$, l'homomorphisme $j^{*}: H^{2 m-q-2}(V, C) \rightarrow H^{2 m-q-2}(S, C)$ est un isomorphisme; il en est donc de même de $j: H_{2 m-q-2}(S, C) \rightarrow H_{2 m-q-2}(V, C)$ et de $a$.

(b) $q=m-1$, l'homomorphisme $j^{*}: H^{m-1}(V, C) \rightarrow H^{m-1}(S, C)$ est un monomorphisme, donc $j$ et $a$ sont des épimorphismes.

(2) Supposons $q+2<m$. Pour toute variété riemannienne compacte $X$, désignons par $\mathfrak{H}^{t}(X, C)$ l'espace vectoriel des formes harmoniques de degré $t$, il est canoniquement isomorphe à $H^{t}(X, C)$. Soient $J$ la forme fondamentale de la variété kählérienne $V$ et $J_{S}$ sa restriction à $S$; alors, la forme différentielle associée à la métrique kählérienne induite sur $S$ par la métrique de $V$ est $J_{S}$. 
On sait que l'application $\epsilon: \mathfrak{F}^{q+2}(V, C) \rightarrow \mathfrak{H}^{2 m-q-2}(V, C)$ qui envoie toute $(q+2)$-forme harmonique $\psi^{q+2}$ de $V$ sur $J^{m-q-2} \wedge \psi^{q+2}$ est un isomorphisme; de même, l'application $\epsilon_{S}: \mathfrak{H}^{q+2}(S, C) \rightarrow \mathfrak{H}^{2 m-q-2}(S, C)$ qui envoie toute $(q+2)$-forme harmonique $\phi^{q+2}$ de $S$ sur $J_{S}^{m-q-2} \wedge \phi^{q+2}$ est un épimorphisme dont le noyau est constitué par les $(q+2)$-formes harmoniques effectives. Désignons par $b^{n}$ l'homomorphisme $\operatorname{JC}^{n}(V, C) \rightarrow \operatorname{JC}^{n}(S, C)$ qui applique les formes harmoniques de $V$ sur la composante harmonique de leur restriction à $S$.

Montrons que le diagramme suivant est commutatif:

$$
\begin{aligned}
& H^{2 m-q-2}(V, C) \approx \mathcal{C}^{2 m-q-2}(V, C) \stackrel{\epsilon}{\approx} \mathcal{F}^{q+2}(V, C) \approx H^{q+2}(V, C) \\
& j^{*} \downarrow \quad Q_{0} b^{2 m-q-2} \downarrow \quad Q_{1} b^{q+2} \downarrow \quad Q_{0} \quad j_{0}^{*} \downarrow \\
& H^{2 m-q-2}(S, C) \underset{\rightrightarrows}{\approx} \mathcal{K}^{2 m-q-2}(S, C) \stackrel{\epsilon_{S}}{\leftarrow} \mathcal{K}^{q+2}(S, C) \approx H^{q+2}(S, C) \text {. }
\end{aligned}
$$

Pour établir la commutativité de $Q$ et celle de $Q_{0}$, montrons que, pour tout entier $n$, le carré

$$
\begin{aligned}
& H^{n}(V, C) \underset{\rightrightarrows}{\rightrightarrows} \mathcal{F}^{n}(V, C) \\
& Q^{n} \quad j^{n} \downarrow \quad b^{n} \downarrow \\
& H^{n}(S, C) \underset{\rightrightarrows}{\rightrightarrows} \mathcal{F}^{n}(S, C)
\end{aligned}
$$

dans lequel $j^{n}$ est induit par l'inclusion de $S$ dans $V$ est commutatif.

Désignons par $B^{t}, Q_{S}^{t}$ (resp. $\mathcal{E}^{t}, \mathcal{E}_{S}^{t}$ ) le faisceau des germes de formes différentielles (resp. de formes différentielles fermées) de degré $t$ différentiables sur $V, S$; l'inclusion de $S$ dans $V$ induit l'homomorphisme $j^{\prime}: \mathbb{B}^{t} \rightarrow \mathbb{B}_{S}^{t}$ tel que le diagramme suivant, où $j^{\prime \prime}$ désigne l'injection, soit commutatif:

$$
\begin{aligned}
0 & \rightarrow \mathcal{E}^{t} \stackrel{j^{\prime \prime}}{\rightarrow} B^{t} \stackrel{d}{\rightarrow} \mathcal{E}^{t+1} \rightarrow 0 \\
& j^{\prime} \downarrow \stackrel{j^{\prime} \downarrow}{i^{\prime} \downarrow} \\
0 & \rightarrow \mathcal{E}_{S}^{t} \stackrel{j^{\prime \prime}}{\rightarrow} \otimes_{S}^{t} \stackrel{d_{S}}{\rightarrow} \varepsilon_{S}^{t+1} \rightarrow 0,
\end{aligned}
$$

il en résulte les diagrammes commutatifs:

$$
\begin{array}{ll}
\quad H^{r}\left(V, \varepsilon^{t+1}\right) & \stackrel{\chi^{r}}{\longrightarrow} H^{r+1}\left(V, \varepsilon^{t}\right) \\
j^{\prime r} \downarrow & j^{\prime r+1} \downarrow \\
H^{r}\left(S, \varepsilon_{S}^{t+1}\right) & \stackrel{\chi^{\prime r}}{\longrightarrow} H^{r+1}\left(S, \varepsilon_{S}^{t}\right), \text { pour } r, t \geq 0, r+t=n
\end{array}
$$

et

$$
\begin{aligned}
& H^{0}\left(V, B^{n-1}\right) \rightarrow H^{0}\left(V, \mathcal{E}^{n}\right) \\
& \downarrow \\
& H^{0}\left(S, \otimes_{S}^{n-1}\right) \rightarrow H^{0}\left(S, \varepsilon_{S}^{n}\right) .
\end{aligned}
$$


D'où le diagramme commutatif:

$$
\begin{aligned}
H^{n}(V, C) & \rightarrow K^{0, n}(V, C) \\
\left(Q^{\prime}\right) \quad j^{n} \downarrow & b^{\prime} \downarrow \\
H^{n}(S, C) & \rightarrow K^{0, n}(S, C),
\end{aligned}
$$

où les homomorphismes horizontaux sont les isomorphismes du théorème de

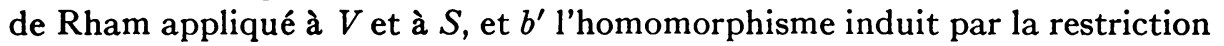
à $S$ des $n$-formes de $V$.

Soient $\phi$ une $n$-forme fermée sur $V, \phi_{S}$ sa restriction à $S$, et soient $H$ (resp. $H_{S}$ ) l'opérateur harmonique sur $V$ (resp. $S$ ) et $d_{S}$ l'opérateur de différentiation sur $S$; on a: $\phi=H \phi+d \psi$ et $(H \phi)_{S}=H_{S}(H \phi)_{S}+d_{S} \xi$ où $\psi$ (resp. $\xi$ ) est une $(n-1)$-forme différentielle sur $V$ (resp. $S)$. Alors $\phi_{S}=(H \phi)_{S}+d_{S} \psi_{S}$ $=H_{S}\left(H \phi_{S}\right)+d_{S}\left(\psi_{S}+\xi\right)$, donc $H_{S} \phi_{S}=H_{S}(H \phi)_{S}$ et le diagramme suivant, dans lequel les isomorphismes horizontaux appliquent toute classe de $n$-formes fermées sur la composante harmonique commune des formes de la classe, est commutatif :

$$
\begin{aligned}
& K^{0, n}(V, C) \approx \mathcal{F}^{n}(V, C) \\
& \left(Q^{\prime \prime}\right) \quad b^{\prime} \downarrow \quad b^{n} \downarrow \\
& K^{0, n}(S, C) \underset{\rightrightarrows}{\rightrightarrows} \mathcal{C}^{n}(S, C) \text {. }
\end{aligned}
$$

La commutativité des carrés $Q^{\prime}$ et $Q^{\prime \prime}$ entraîne celle de $Q^{n}$.

Soit maintenant $\phi$ une $(q+2)$-forme harmonique sur $V$, on a:

$$
b^{q+2} \phi=H_{S} \phi_{S} ; \quad \epsilon_{S} b^{q+2} \phi=J_{S}^{2 m-q-2} \wedge H_{S} \phi_{S} .
$$

D'autre part:

$$
\epsilon \phi=J^{2 m-q-2} \wedge \phi ; b^{2 m-q-2} \epsilon \phi=H_{S}\left(J^{2 m-q-2} \wedge \phi\right)_{S}=H_{S}\left(J_{S}^{2 m-q-2} \wedge \phi_{S}\right) ;
$$

il existe une forme $\xi^{\prime}$ sur $S$ telle que $\phi_{S}=H_{S} \phi_{S}+d_{S} \xi^{\prime}$, alors:

$$
\begin{aligned}
\left(J^{2 m-q-2} \wedge \phi\right)_{S} & =J_{S}^{2 m-q-2} \wedge \phi_{S}=J_{S}^{2 m-q-2} \wedge\left(H_{S} \phi_{S}+d_{S} \xi^{\prime}\right) \\
& =J_{S}^{2 m-q-2} \wedge H_{S} \phi_{S}+d_{S}\left(J_{S}^{2 m-q-2} \wedge \xi^{\prime}\right) ;
\end{aligned}
$$

donc $H_{S}\left(J^{2 m-q-2} \wedge \phi\right)_{S}=J_{S}^{2 m-q-2} \wedge H_{S} \phi_{S}$, ce qui prouve $\epsilon_{S} b^{q-2}=b^{2 m-q-2} \epsilon$ : le carré $Q_{1}$ est commutatif.

$\mathrm{Si}\{S\}$ est suffisamment ample au sens de Kodaira, d'après le Théorème 2 de [4], pour $q+2<m-1, j_{0}^{*}$ est un isomorphisme, alors $j^{*}$ est un épimorphisme, donc $j$ et $a$ sont des monomorphismes, c.q.f.d.

Proposition 7. Si $V$ est une variêté kählérienne compacte, $S$ un ensemble analytique principal sans singularité de $V$, et si $\{S\}$ est suffisamment ample, alors: 
pour $p+q \geqq m+1$ ou $p+q<m-3$, ou $p=m$ et $q=0$, on $a$ :

$$
\operatorname{dim} H^{q}\left(V, \mathcal{Q}_{S}^{p}\right)=\operatorname{dim} H^{p-1, q}(S, C)+\operatorname{dim} H^{q}\left(S, \Omega^{p}\left(\{S\}_{S}\right)\right) ;
$$

pour $p+q=m$ et $q>0$, on $a$ :

$$
\operatorname{dim} H^{m-p}\left(V, \mathcal{R}_{S}^{p}\right)=\operatorname{dim} H^{p, m-p+1}(V, C)+\operatorname{dim} H^{m-p}\left(S, \Omega^{p}\left(\{S\}_{S}\right)\right) .
$$

Démonstration. (a) Le faisceau $M_{S}^{p}$ étant isomorphe à $\Omega^{p}(\{S\})$, si $\{S\}$ est suffisamment ample, on a: $H^{q}\left(V, M_{S}^{p}\right)=0$ pour $q \geqq 1$, d'après le Théorème 1 de [3], donc l'homomorphisme $H^{t}\left(V, \Theta_{S}^{p}\right) \rightarrow H^{t+1}\left(V, \Omega^{\prime \prime} p(\{S\})\right)$ défini par la suite exacte $\left(5^{\prime}\right) \quad 0 \rightarrow{\Omega^{\prime \prime} p}^{p}(\{S\}) \rightarrow M_{S}^{p} \rightarrow Q_{S}^{p} \rightarrow 0$, est un épimorphisme, il en résulte que, dans (16), on $\mathrm{a}$ :

conoyau $\left[H^{t}\left(V, \mathcal{R}_{S}^{p}\right) \rightarrow H^{t}\left(V, Q_{S}^{p}\right)\right] \approx \operatorname{noyau}\left[H^{t+1}\left(V, \Omega^{p-1}[S]\right) \stackrel{k}{\rightarrow} H^{t+2}\left(V, \Omega^{p}\right)\right] ;$ ce dernier espace est isomorphe au noyau de $k^{\prime}: H^{p-1, t+1}(S, C) \rightarrow H^{p, t+2}(V, C)$, d'après la définition de $k^{\prime}$.

(b) $S$ étant kählérienne compacte, il existe, dans chaque classe de $H^{p-1, t+1}(S, C)$, une forme $\omega$ harmonique sur $S$, donc $d$-fermée. L'homomor phisme $k^{\prime}$ (qui figure dans le Lemme 5) peut alors être défini par l'application des $(p-1, t+1)$-formes $d$-fermées $\omega$ de $S$ sur les courants $\omega^{\prime}, d$-fermés, de type $(p, t+2)$ de $V$ tels que $\omega^{\prime}[\psi]=2 \pi i \int_{s} \omega \wedge \psi$. Donc $k^{\prime}$ est la restriction aux classes de formes de type $(p-1, t+1)$ de l'homomorphisme $a\left({ }^{1}\right)$ du Lemme 6. Enfin, $a$ étant un homomor phisme de type $(1,1)$, l'image de $k^{\prime}$ est la partie de l'image de $a\left({ }^{1}\right)$ constituée par les classes de type $(p, t+2)$. Alors, d'après le Lemme 6:

si $p+t<m-3$ ou $p+t \geqq m, k^{\prime}$ est un monomorphisme, donc le noyau de $k^{\prime}$ est nul, d'où d'après $(\mathrm{a}):$ conoyau $\left[H^{t}\left(V, \Re_{S}^{p}\right) \rightarrow H^{t}\left(V, \Theta_{S}^{p}\right)=0\right.$;

si $p+t=m-1, k^{\prime}$ est un épimorphisme, donc la dimension du noyau de $\quad k^{\prime}$ est: $\operatorname{dim} H^{p-1, m-p}(S, C)-\operatorname{dim} H^{p, m-p+1}(V, C)=\operatorname{dim}$ conoyau $\left[H^{m-p-1}\left(V, \mathcal{R}_{S}^{p}\right) \rightarrow H^{m-p-1}\left(V, \Theta_{S}^{p}\right)\right]$.

(c) La suite exacte (14) entraîne:

$\operatorname{dim} \boldsymbol{H}^{\boldsymbol{q}}\left(V, \mathfrak{R}_{S}^{p}\right)=\operatorname{dim} H^{q}\left(V, \Omega^{p-1}[S]\right)+\operatorname{dim} H^{q}\left(V, Q_{S}^{p}\right)-\operatorname{dim}$ conoyau $\left[H^{q-1}\left(V, \mathfrak{Q}_{S}^{p}\right)\right.$

$$
\left.\rightarrow H^{q-1}\left(V, Q_{S}^{p}\right)\right]-\operatorname{dim} \text { conoyau }\left[H^{q}\left(V, Q_{S}^{p}\right) \rightarrow H^{q}\left(V, Q_{S}^{p}\right)\right] \text {. }
$$

D'après les isomorphismes

$$
H^{q}\left(V, \Omega^{p-1}[S]\right) \approx H^{q}\left(S, \Omega^{p-1}[S]\right) \approx K^{p-1, q}(S, C),
$$

et

(1) Au produit pres par $2 \pi i$. 


$$
H^{q}\left(V, Q_{S}^{p}\right) \approx H^{q}\left(V, \Omega^{p}\left(\{S\}_{S}\right)\right) \approx H^{q}\left(S, \Omega^{p}\left(\{S\}_{S}\right)\right),
$$

on a: $\operatorname{dim} H^{q}\left(V, \Omega^{p-1}[S]\right)=\operatorname{dim} H^{p-1, q}(S, C)$, et $\operatorname{dim} H^{q}\left(V, Q_{S}^{p}\right)$ $=\operatorname{dim} H^{q}\left(S, \Omega^{p}\left(\{S\}_{S}\right)\right)$.

Alors, d'après (b),

si $p+q-1 \geqq m$ ou $p+q<m-3$, ou $p=m$ et $q=0$, on a:

$$
\operatorname{dim} H^{q}\left(V, \boldsymbol{R}_{S}^{p}\right)=\operatorname{dim} H^{p-1, q}(S, C)+\operatorname{dim} H^{q}\left(S, \Omega^{p}\left(\{S\}_{S}\right)\right) ;
$$

si $p+q=m$ et $q>0$, on a:

$\operatorname{dim} H^{m-p}\left(V, \mathcal{R}_{S}^{p}\right)=\operatorname{dim} H^{p-1, m-p}(S, C)+\operatorname{dim} H^{m-p}\left(S, \grave{\Omega}^{p}\left(\{S\}_{S}\right)\right)$

$$
-\operatorname{dim} H^{p-1, m-p}(S, C)+\operatorname{dim} H^{p, m-p+1}(V, C), \quad \text { c.q.f.d. }
$$

\section{Cohomologie à coefficients dans $\delta_{S}^{p}$.}

Proposition 8. Si l'ensemble analytique principal $S$ de $V$ est une variété kählérienne compacte, on $a$ :

pour $p>0$ :

$$
\operatorname{dim} H^{q}\left(V, s_{S}^{p}\right)=\operatorname{dim} K^{p, q}(S, C)+\operatorname{dim} H^{q}\left(S, \Omega^{p}\left(\{S\}_{S}\right)\right) ;
$$

pour $p=0$ :

$$
\operatorname{dim} H^{q}\left(V, s_{S}^{0}\right)=\operatorname{dim} H^{q}\left(S, \Omega^{0}\left(\{S\}_{S}\right)\right)
$$

Démonstration. Pour $q>0$, la suite exacte

$$
0 \rightarrow E^{p}[S] \stackrel{\lambda^{\prime}}{\rightarrow} S_{S}^{p} \stackrel{h^{\prime}}{\rightarrow} Q_{S}^{p} \rightarrow 0
$$

définit la suite exacte de cohomologie:

$$
\begin{aligned}
\cdots \rightarrow H^{q-1}\left(V, Q_{S}^{p}\right) \stackrel{\beta^{q-1}}{\longrightarrow} H^{q}\left(V, E^{p}[S]\right) \rightarrow H^{q}\left(V, s_{S}^{p}\right) \rightarrow H^{q}\left(V, Q_{S}^{p}\right) \\
\stackrel{\beta^{q}}{\rightarrow} H^{q+1}\left(V, E^{p}[S]\right) \rightarrow \cdots
\end{aligned}
$$

Considérons le diagramme commutatif suivant qui se déduit de (13) et de (11):

$$
\begin{aligned}
0 & \rightarrow \Omega^{p-1}[S] \stackrel{\lambda}{\rightarrow} R_{S}^{p} \stackrel{h}{\rightarrow} Q_{S}^{p} \rightarrow 0 \\
d \downarrow \quad & -d^{\prime} \downarrow \quad i \downarrow \\
0 & \rightarrow E^{p}[S] \stackrel{\lambda^{\prime}}{\rightarrow} S_{S}^{p} \stackrel{h^{\prime}}{\rightarrow} Q_{S}^{p} \rightarrow 0 .
\end{aligned}
$$

On en déduit le diagramme de cohomologie: 


$$
\begin{aligned}
& H^{t}\left(V, Q_{S}^{p}\right) \stackrel{\gamma}{\rightarrow} H^{t+1}\left(V, \Omega^{p-1}[S]\right) \approx H^{t+1}\left(S, \Omega^{p-1}[S]\right)
\end{aligned}
$$

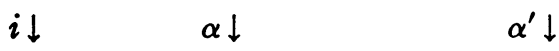

$$
\begin{aligned}
& H^{t}\left(V, Q_{S}^{p}\right) \stackrel{\beta^{t}}{\rightarrow} H^{t+1}\left(V, E^{p}[S]\right) \underset{\rightrightarrows}{\rightarrow} H^{t+1}\left(S, E^{p}[S]\right),
\end{aligned}
$$

pour $t \geqq-1$.

Si $S$ est kählérienne compacte, l'homomorphisme $\alpha^{\prime}$ défini par $d: \Omega^{p-1}[S]$ $\rightarrow E^{p}[S]$ a une image nulle d'après la Proposition 1.13 de [2]; il en est donc de même de $\alpha$; de plus $\beta^{t}=\beta^{t} \circ i=\alpha \circ \gamma$ d'après la commutativité du diagramme (24), donc $\beta^{t}$ a une image nulle. Alors, on déduit de (23) la suite exacte

$$
0 \rightarrow H^{q}\left(V, E^{p}[S]\right) \rightarrow H^{q}\left(V, s_{S}^{p}\right) \rightarrow H^{q}\left(V, Q_{S}^{p}\right) \rightarrow 0
$$

Enfin $S$ étant compacte les espaces $H^{q}\left(V, E^{p}[S]\right) \approx H^{q}\left(S, E^{p}[S]\right)$ $\approx K^{p, q}(S, C)$ et $H^{q}\left(V, \Theta_{S}^{p}\right) \approx H^{q}\left(S, \Omega^{p}\left(\{S\}_{S}\right)\right.$ ) (d'après (21)) sont de dimension finie, donc d'après $\left(23^{\prime}\right)$, on $a$ :

$$
\begin{aligned}
\operatorname{dim} H^{q}\left(V, S_{s}^{p}\right)= & \operatorname{dim} H^{q}\left(V, E^{p}[S]\right)+\operatorname{dim} H^{q}\left(V, Q_{s}^{p}\right)=\operatorname{dim} K^{p, q}(S, C) \\
& +\operatorname{dim} H^{q}\left(S, \Omega^{p}\left(\{S\}_{S}\right)\right) .
\end{aligned}
$$

Pour $p=0$, on a: $\delta_{S}^{0} \approx \Omega^{0}(\{S\} s)$, d'apres (12) et (7), d'où: $\operatorname{dim} H^{a}\left(V, \delta_{s}^{0}\right)$ $=\operatorname{dim} H^{q}\left(S, \Omega^{0}\left(\{S\}_{s}\right)\right)$.

\section{FORMES MÉROMORPHES LOCALEMENT EXACTES}

6. Généralités. Soit $M^{p}$ le faisceau des germes de $p$-formes méromorphes sur $V$.

DÉfINITION 3. On appelle $(p+1)$-forme méromorphe localement exacte toute section du faisceau $d M^{p}$.

Proposition 9. Le sous-faisceau de $d M^{p}$ constitué par les germes admettant l'ensemble analytique principal sans singularité $S$ comme ensemble polaire d la multiplicité deux au plus est le faisceau $d M_{S}^{p}$.

Démonstration. Soit, en un point $x \in V$, un élément $\sigma=d \chi$ de $\left(d M^{p}\right)_{x}$ admettant le germe $S_{x}$ défini par $S$ en $x$, comme ensemble polaire à la multiplicité deux au plus. $S$ étant sans singularité, il existe un système de coordonnées $(w, y)$ en $x$, dans lequel $S_{x}=[w]$; de plus, $\sigma$ étant un germe de $(p+1)$-forme méromorphe fermée, d'après la Proposition 3.2 de [2], il existe un germe de $p$-forme holomorphe $B$ et un germe de $p$-forme holomorphe fermée $A$ tels que $\sigma=d \chi=d(B / w)+(d w / w) \wedge A$.

(1) $p=0$, alors $A$ est une constante; comme $d w / w$ n'est pas la différentielle d'une fonction méromorphe, on a $A=0$, donc $\sigma=d \chi^{\prime}$ avec $\chi^{\prime}=B / w$. 
(2) $p>0$, alors $A$ étant fermé, il existe un germe de $(p-1)$-forme holomorphe $a$ tel que $A=d a$, donc $\sigma=d \chi^{\prime}$ avec $\chi^{\prime}=B / w-(d w / w) \wedge a$.

Dans les deux cas, on voit qu'il existe $\chi^{\prime} \in M_{S}^{p}$ tel que $\sigma=d \chi^{\prime}$, c.q.f.d.

Corollaire 10. $H^{0}\left(V, d M_{S}^{p}\right)$ est l'espace des $(p+1)$-formes méromorphes localement exactes dont l'ensemble polaire est $S$ à la multiplicité deux au plus.

Lemme 11. Considérons le diagramme commutatif:

$$
\begin{array}{ll}
H^{q}\left(V, M_{S}^{p}\right) \stackrel{\delta}{\rightarrow} & H^{q}\left(V, d M_{S}^{p}\right) \\
j \downarrow & j^{\prime} \downarrow \\
H^{q}\left(V, M^{p}\right) \stackrel{\delta^{\prime}}{\rightarrow} H^{q}\left(V, d M^{p}\right)
\end{array}
$$

dans lequel $j$ et $j^{\prime}$ désignent les homomorphismes induits par les injections $M_{S}^{p} \rightarrow M^{p}$ et $d M_{S}^{p} \rightarrow d M^{p} ; \delta$ et $\delta^{\prime}$ les homomorphismes induits par $d$, et soit $\omega$ un élément de $H^{q}\left(V, d M^{p}\right)$ appartenant a l'intersection des images de $\delta^{\prime}$ et de $j^{\prime}$. Alors:

(1) si $p=0, \omega$ appartient a l'image de $j^{\prime} \circ \delta$;

(2) si $p>0, \omega n$ nappartient pas nécessairement à l'image de $j^{\prime} \circ \delta$.

Démonstration. (1) $p=0$. Soit $\bar{\omega} \in H^{q}\left(V, M^{0}\right)$ tel que $\omega=\delta^{\prime} \bar{\omega}$. Considérons un recouvrement ouvert $r=\left\{U_{i}\right\}$ de $V$ suffisamment fin pour qu'il existe un $q$-cocycle $\left\{\omega_{i_{0}} \ldots i_{q}\right\}$ (resp. $\left.\left\{\bar{\omega}_{i_{0}} \ldots i_{q}\right\}\right)$ du nerf $N_{r}$ de $r$ dont l'image canonique de la classe de cohomologie dans $H^{q}\left(V, d M_{S}^{0}\right)$ (resp. dans $\left.H^{0}\left(V, M^{0}\right)\right)$ soit $\omega$ (resp. $\bar{\omega}$ ). Désignons par $T$ le complémentaire, supposé non vide, de $S_{i_{0} \cdots i_{q}}=S \cap U_{i_{0}} \cap \cdots \cap U_{i_{q}}$ dans l'ensemble polaire de la fonction $\bar{\omega}_{i_{0}} \cdots i_{q}$. En un point $x$ non singulier de $T$ et n'appartenant pas à $S_{i_{0}} \cdots i_{q}$, soit [w] le germe analytique défini par $T$, il existe un entier $n>0$ et un germe holomorphe $\xi$ non nul pour $w=0$, tels que $\bar{\omega}_{i_{0}} \cdots_{i_{q}}=\xi / w^{n}$; alors $d \bar{\omega}_{i_{0}} \cdots i_{q}=\left(d \xi / w^{n}\right)$ $-n\left(d w / w^{n+1}\right) \xi$, en $x$; il en résulte que $d \bar{\omega}_{i_{0}} \ldots i_{q}=\omega_{i_{0}} \ldots i_{q}$ admet tous les points simples de $T-T \cap S_{i_{0}} \cdots_{i_{q}}$ comme pôles à la multiplicité $n+1$, donc $\omega_{i_{0}} \cdots i_{q}$ admet $T$ tout entier comme ensemble polaire à la multiplicité $n+1$, ce qui est impossible; donc $T=\varnothing$.

Soient maintenant $x \in S_{i_{0}} \ldots i_{q} ;[w]$ le germe analytique défini par $S$ en $x$ et $\xi$ un germe holomorphe non nul en $x$ pour $w=0$, tels que $\bar{\omega}=\xi / w^{n}$ en $x$. Comme $d \bar{\omega} \in\left(d M_{S}^{p}\right)_{x}$, on a $n \leqq 1$, ce qui achève la démonstration de (1). (2) $p>0$. Le Corollaire 3.15 de [2] fournit, pour $q=0$ et $p=1$, un exemple de forme $\bar{\omega}$ sur $V$ dont l'ensemble polaire est non vide, tandis que $d \bar{\omega}$ est holomorphe, ce qui établit (2).

Considérons la suite exacte de faisceaux:

$$
0 \rightarrow m_{S}^{p} \rightarrow M_{S}^{p} \rightarrow d M_{S}^{p} \rightarrow 0 ;
$$

elle définit la suite exacte de cohomologie: 


$$
\cdots \rightarrow H^{q-1}\left(V, d M_{S}^{p}\right) \rightarrow H^{q}\left(V, m_{S}^{p}\right) \rightarrow H^{q}\left(V, M_{S}^{p}\right) \rightarrow H^{q}\left(V, d M_{S}^{p}\right) \rightarrow \cdots
$$

On en déduit les suites exactes:

$$
0 \rightarrow H^{0}\left(V, m_{S}^{p}\right) \rightarrow H^{0}\left(V, M_{S}^{p}\right) \rightarrow H^{0}\left(V, d M_{S}^{p}\right) \rightarrow \mathcal{E}_{S}^{p+1,0} \rightarrow 0,
$$

$$
0 \rightarrow \varepsilon_{S}^{p+1, q-1} \rightarrow H^{q}\left(V, m_{S}^{p}\right) \rightarrow H^{q}\left(V, M_{S}^{p}\right) \rightarrow H^{q}\left(V, d M_{S}^{p}\right) \rightarrow \varepsilon_{S}^{p+1, q} \rightarrow 0,
$$

avec $\mathcal{E}^{p+1, t}=$ conoyau $\left[H^{t}\left(V, M_{S}^{p}\right) \rightarrow H^{t}\left(V, d M_{S}^{p}\right)\right]$.

Du Corollaire 10 et du Lemme 11 résulte:

Proposition 12. Dans les notations ci-dessus:

(a) L'espace vectoriel $\mathcal{E}_{S}^{1,0}$ est l'espace des classes de 1-formes méromorphes localement exactes dont l'ensemble polaire est $S$ a la multiplicité deux au plus, modulo les différentielles de fonctions méromorphes sur $V$.

(b) L'espace vectoriel $\varepsilon_{S}^{p+1,0}$ est l'espace des classes de $(p+1)$-formes méromorphes localement exactes dont l'ensemble polaire est $S$ a la multiplicité doux au plus, modulo les différentielles de formes méromorphes sur $V$ dont l'ensemble polaire est $S$ à la multiplicité un au plus.

On se propose de calculer $\operatorname{dim} \varepsilon_{S}^{p+1,0}$ et plus généralement $\operatorname{dim} \varepsilon_{S}^{p+1, t}$ dans certains cas particuliers où les espaces vectoriels figurant dans les suites $(26)_{q}(0 \leqq q \leqq t)$ sont de dimension finie.

On a la formule de récurrence déduite de $(26)_{q}$ :

$$
\begin{aligned}
\operatorname{dim} \varepsilon_{S}^{p+1, q-1}+\operatorname{dim} \varepsilon_{S}^{p+1, q}= & \operatorname{dim} H^{q}\left(V, m_{S}^{p}\right)-\operatorname{dim} H^{q}\left(V, M_{S}^{p}\right) \\
& +\operatorname{dim} H^{q}\left(V, d M_{S}^{p}\right) .
\end{aligned}
$$

On va évaluer les termes du second membre.

Les suites exactes (2), (9) et (10) définissent respectivement les suites exactes:

$$
\begin{aligned}
& 0 \rightarrow k_{1}^{p, q} \rightarrow H^{q}\left(V, M_{S}^{p}\right) \rightarrow H^{q}\left(V, Q_{S}^{p}\right) \rightarrow H^{q+1}\left(V, \Omega^{p}\right) \rightarrow k_{1}^{p, q+1} \rightarrow 0, \\
& \text { avec } \quad k_{1}^{p, t+1}=\text { conoyau }\left[H^{t}\left(V, \Omega_{S}^{p}\right) \rightarrow H^{t+1}\left(V, \Omega^{p}\right)\right] ; \\
& 0 \rightarrow k_{2}^{p, q} \rightarrow H^{q}\left(V, m_{S}^{p}\right) \rightarrow H^{q}\left(V, r_{S}^{p}\right) \rightarrow H^{q+1}\left(V, E^{p}\right) \rightarrow k_{2}^{p, q+1} \rightarrow 0, \\
& \text { avec } \quad k_{2}^{p, t+1}=\text { conoyau }\left[H^{t}\left(V, r_{S}^{p}\right) \rightarrow H^{t+1}\left(V, E^{p}\right)\right] ; \\
& 0 \rightarrow k_{3}^{p, q} \rightarrow H^{q}\left(V, d M_{S}^{p}\right) \rightarrow H^{q}\left(V, S_{S}^{p}\right) \rightarrow H^{q+1}\left(V, E^{p+1}\right) \rightarrow k_{3}^{p, q+1} \rightarrow 0, \\
& \text { avec } \quad k_{3}^{p, t+1}=\text { conoyau }\left[H^{t}\left(V, S_{S}^{p}\right) \rightarrow H^{t+1}\left(V, E^{p+1}\right)\right] .
\end{aligned}
$$

On a évalué, au $\S I$, au moins dans des cas particuliers, les dimensions 
des espaces $H^{q}\left(V, \mathcal{R}_{S}^{p}\right), H^{q}\left(V, r_{S}^{p}\right)$ et $H^{q}\left(V, s_{S}^{p}\right)$; le problème est donc ramené à l'évaluation des dimensions des espaces $k_{1}^{p, t+1}, k_{2}^{p, t+1}, k_{3}^{p, t+1}$, pour $t \geqq 0$.

7. Les espaces auxiliaires $k_{1}^{p, t+1}, k_{2}^{p, t+1}$ et $k_{3}^{p, t+1}$.

Proposition 13. Si $V$ est kählérienne compacte, si $S$ est un ensemble analytique principal sans singularitê, et si $\{S\}$ est suffisamment ample, on a $k_{1}^{p, t+1}=0$, pour $t \geqq 0$.

Démonstration. Considérons la suite exacte de cohomologie déduite de (2):

$$
\cdots \rightarrow H^{t}\left(V, \Omega_{S}^{p}\right) \rightarrow H^{t+1}\left(V, \Omega^{p}\right) \stackrel{\lambda}{\rightarrow} H^{t+1}\left(V, M_{S}^{p}\right) \rightarrow \cdots .
$$

Le faisceau $M_{S}^{p}$ est isomorphe à $\Omega^{p}(\{S\})$, et si $V$ est kählérienne compacte et $\{S\}$ suffisamment ample, on a $H^{t+1}\left(V, \Omega^{p}(\{S\})\right)=0$ pour $t \geqq 0$, d'après le Théorème 1 de [3], il en résulte que l'image de $\lambda$ est nulle, donc $k_{1}^{p, t+1}=0$.

Calcul de $\operatorname{dim} k_{2}^{p, t+1}$. Soit $\mu^{t}$ l'homomorphisme $H^{t}\left(V, r_{S}^{p}\right) \rightarrow H^{t+1}\left(V, E^{p}\right)$ de la suite exacte (28). Désignons par $\nu: E^{p-1}[S] \rightarrow r_{S}^{p}$ l'isomorphisme de la Proposition 2, par $\nu^{\prime t}$ l'isomorphisme $H^{t}\left(S, E^{p-1}[S]\right) \rightarrow H^{t}\left(V, r_{S}^{p}\right)$ défini par $\nu$ et $\operatorname{par} \delta^{t+1}$ (resp. $\left.\delta_{S}^{t}\right)$ l'isomorphisme $K^{p, t+1}(V, C) \rightarrow H^{t+1}\left(V, E^{p}\right)$ (resp. $\left.K^{p-1, t}(S, C) \rightarrow H^{t}\left(S, E^{p-1}[S]\right)\right)$ du Théorème 1.6 de [2].

Lemme 14. Dans les notations ci-dessus, l'homomorphisme $h^{t+1}$ $=\left(\delta^{t+1}\right)^{-1} \circ \mu^{t} \circ \nu^{\prime t} \circ \delta_{S}^{t}: K^{p-1, t}(S, C) \rightarrow K^{p, t+1}(V, C)$ est défini par l'application de la classe de d-cohomologie de toute $(p+t-1)$-forme $d$-fermée $\omega$ de $S$, de premier degré au moins égal a $(p-1)$ sur la classe de d-cohomologie du courant $d$-fermé de $V$, de degrê $(p+t+1)$, de premier degré au moins égal à $p$, défini par:

$$
\omega^{\prime}[\psi]=2 \pi i \int_{S} \omega \wedge \psi
$$

Démonstration. Dans les notations du $\mathrm{n}^{\circ} 2$, l'homomorphisme $\nu$ est défini ainsi: pour $x \in U_{j}$, si $\eta^{\prime \prime} \in\left(E^{p-1}[S]\right)_{x}$, le germe de courant $\nu \eta^{\prime \prime}$ est $d^{\prime \prime}\langle\tau\rangle$ tel que:

$$
d^{\prime \prime}\langle\tau\rangle[\psi]=2 \pi i \int_{S} \eta_{i}^{\prime \prime} \wedge \psi
$$

Considérons l'homomorphisme $\nu^{\prime}$. Soit $\gamma$ un élément de $H^{t}\left(S, E^{p-1}[S]\right)$, il existe un recouvrement $r=\left\{U_{j}\right\}$ et un $t$-cocycle $\left\{\eta_{j_{0}}^{\prime \prime} \ldots j_{t}\right\}$ de $N_{r}$ à coefficients dans $E^{p-1}[S]$ dont la classe de cohomologie a pour image canonique $\gamma$. Si $r$ est assez fin $\alpha=\nu^{\prime} \gamma$ est l'image canonique, dans $H^{t}\left(V, r_{S}^{p}\right)$, de la classe de cohomologie du cocycle $\left\{d^{\prime \prime}\left\langle\tau_{j_{0}} \ldots j_{t}\right\rangle\right\}$ de $N_{r}$ à coefficients dans $r_{S}^{p}$, le courant $d^{\prime \prime}\left\langle\tau_{j_{0}} \cdots j_{t}\right\rangle$ étant défini par la formule (30) sur $U_{j_{0}} \cap \cdots \cap U_{j_{t}} \neq \varnothing$. 
Considérons l'homomorphisme $\mu^{t}$. Soit $\alpha \in H^{t}\left(V, r_{S}^{p}\right)$. Pour $r$ assez fin, il existe $\tau_{j_{0}} \ldots j_{t} \in \Gamma\left(U_{j_{0}} \cap \cdots \cap U_{j_{t}}, m_{S}^{p}\right)$ tel que $\alpha$ soit l'image canonique de la classe de cohomologie du cocycle $\left\{d^{\prime \prime}\left\langle\tau_{j_{0}} \cdots j_{t}\right\rangle\right\}$, on a:

$$
\begin{aligned}
& \sum_{l=0}^{t+1}(-1)^{l} d^{\prime \prime}\left\langle\tau_{i_{0}} \ldots i_{l} \ldots t_{t+1}\right\rangle=0 \text { sur } U_{i_{0}} \cap \cdots \cap U_{i_{t+1}} \neq \varnothing, \text { donc } \\
& \sum_{l=0}^{t+1}(-1)^{l} \tau_{i_{0}} \ldots t_{l} \cdots i_{t+1}=e_{i_{0}} \cdots i_{t+1} \in \Gamma\left(U_{i_{0}} \cap \cdots \cap U_{i_{t+1}}, E^{p}\right) .
\end{aligned}
$$

Par définition de $\mu^{t}$, l'image canonique de la classe de cohomologie du $(t+1)$ cocycle $\left\{e_{i_{0}} \ldots i_{t+1}\right\}$ dans $H^{t+1}\left(V, E^{p}\right)$ est $\alpha ; r_{S}^{p}$ est un sous-faisceau du faisceau $E^{p, 1}$ des germes de $(p+1)$-formes fermées dont le premier degré est au moins

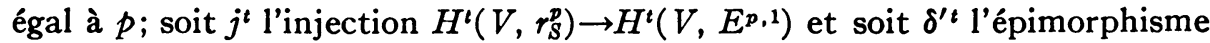
$H^{t}\left(V, E^{p, 1}\right) \rightarrow H^{t+1}\left(V, E^{p}\right)$ qui figure dans la démonstration du Théorème 1.6 de [2]. Le diagramme

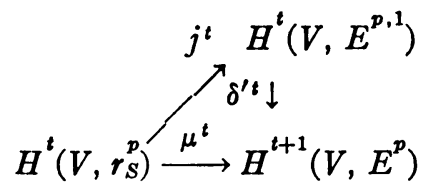

est commutatif car $m_{S}^{p}$ est un sous-faisceau du faisceau fin $B^{p, 0}$ des germes de courants de type $(p, 0)$ et, d'après (31), pour $\alpha \in H^{t}\left(V, r_{S}^{p}\right), \mu^{t} \alpha$ est l'image, par $\delta^{\prime t}$, de l'image canonique, dans $H^{t}\left(V, E^{p, 1}\right)$ de la classe de cohomologie du cocycle $\left\{d^{\prime \prime}\left\langle\tau_{j_{0} \cdots j_{t}}\right\rangle\right\}$.

Si $t=0$, d'après la démonstration du Théorème 1.6 de [2], $h^{1}$ est l'homomorphisme qui associe, à la forme $\omega \in H^{0}\left(S, E^{p-1}[S]\right)$, la classe de $d$-cohomologie du courant $\omega^{\prime} \in H^{0}\left(V, E^{p, 1}\right)$ défini par $\omega^{\prime}[\psi]=2 \pi i \int_{S} \omega \wedge \psi$.

Si $t>0$, on sait que $\delta^{\prime t}$ est un isomorphisme, alors l'homomorphisme $h^{\prime}=\left(\delta^{\prime t}\right)^{-1} \circ \mu^{t} \circ \nu^{\prime t}$ applique l'image canonique de la classe de cohomologie du $t$-cocycle $\left\{\eta_{j_{0} \ldots j_{t}}^{\prime \prime}\right\}$ sur l'image canonique de la classe de cohomologie du cocycle $\left\{\sigma_{j_{0} \ldots j_{t}}\right\}$ où le courant $\sigma_{j_{0} \ldots j_{t}}$ sur $U_{j_{0}} \cap \cdots \cap U_{j_{t}} \neq \varnothing$ est défini par:

$$
\sigma_{j_{0} \cdots i_{t}}[\psi]=2 \pi i \int_{S} \eta_{j_{0} \cdots j_{t}}^{\prime \prime} \wedge \psi
$$

On sait qu'il existe un isomorphisme canonique $\delta^{\prime \prime t}$ tel que $\delta^{t+1}=\delta^{\prime t} \circ \delta^{\prime \prime t}$ et on a:

$$
h^{t+1}=\left(\delta^{\prime \prime} t\right)^{-1} \circ h^{\prime} \circ \delta_{S}^{\prime} \text {. }
$$

Alors, d'après l'interprétation de $h^{r}$ et la démonstration du Théorème 1.6 de [2], il est clair que $h^{t+1}$ est l'homomorphisme qui associe, à la classe de $d$-cohomologie de toute $(p+t-1)$-forme fermée $\omega$ de $S$, de premier degré au moins égal à $(p-1)$, la classe de cohomologie du courant $\omega^{\prime}, d$-fermé de $V$, de degré $(p+t+1)$, de premier degré au moins égal à $p$, défini par: 


$$
\omega^{\prime}[\psi]=2 \pi i \int_{S} \omega \wedge \psi .
$$

Proposition 15. Si $V$ est une variété kählérienne compacte, si $S$ est un ensemble analytique principal sans singularité et si $\{S\}$ est suffisamment ample, alors:

pour $p+t<m-2$ ou $p+t \geqq m+1$, ou $t=m$ et $p=0$, on $a$ :

$$
\operatorname{dim} k_{2}^{p, t+1}=\operatorname{dim} K^{p, t+1}(V, C)-\operatorname{dim} K^{p-1, t}(S, C) ;
$$

pour $p+t=m$ et $p>0$, on $a$ :

$$
\operatorname{dim} k_{2}^{p, m-p+1}=0
$$

Démonstration. Par définition de $h^{t+1}$, l'espace $k_{2}^{p, t+1}$ est isomorphe au conoyau de l'homomorphisme $h^{t+1}: K^{p-1, t}(S, C) \rightarrow K^{p, t+1}(V, C)$.

D'après le Lemme $14, h^{t+1}$ est la restriction de l'homomorphisme $a^{(1)} \mathrm{du}$ Lemme 6 au sous-espace $K^{p-1, t}(S, C)$ de $K^{0, p+t-1}(S, C)$. De plus, $a$ étant de type $(1,1)$ l'image de $h^{t+1}$ est la partie de l'image de $a^{(1)}$ constituée par les classes de degré $p+t+1$ et de premier degré au moins égal à $p$. Alors, d'après le Lemme 6:

si $p+t-1<m-3$ ou $p+t-1 \geqq m, h^{t+1}$ est un monomorphisme, donc:

$$
\operatorname{dim} k_{2}^{p, t+1}=\operatorname{dim} K^{p, t+1}(V, C)-\operatorname{dim} K^{p-1, t}(S, C) ;
$$

si $p+t-1=m-1, h^{t+1}$ est un épimorphisme, donc:

$$
\operatorname{dim} k_{2}^{p, m-p+1}=0,
$$

Calcul de $\operatorname{dim} k_{3}^{p, t+1}$.

Propositron 16. Si $V$ est une variêté kählérienne compacte, $S$ un sousensemble analytique principal sans singularité et si $\{S\}$ est șuffisamment ample, on $a$ :

pour $p>0$ :

$$
\operatorname{dim} k_{3}^{p, t+1}=\operatorname{dim} k_{2}^{p+1, t+1} \text {, i.e.: }
$$

si $p+t<m-3$ ou $p+t \geqq m$, on $a$ :

$$
\operatorname{dim} k_{3}^{p, t+1}=\operatorname{dim} K^{p+1, t+1}(V, C)-\operatorname{dim} K^{p, t}(S, C) ;
$$

si $p+t=m-1$, on $a$ :

$$
\operatorname{dim} k_{3}^{k, m-p}=0
$$

pour $p=0$ :

$$
\operatorname{dim} k_{3}^{0, t+1}=\operatorname{dim} K^{1, t+1}(V, C) .
$$

Démonstration. Nous reprenons les notations du Lemme 14, en faisant 
figurer un second indice déterminé par le degré des éléments du faisceau des coefficients, les homomorphismes utilisés dans le Lemme 14 et la Proposition 15 correspondant au second indice $p$ :

$$
\begin{array}{ll}
\nu^{t, p}: H^{t}\left(S, E^{p-1}[S]\right) & \approx H^{t}\left(V, r_{S}^{p}\right) ; \\
\mu^{t, p}: H^{t}\left(V, r_{S}^{p}\right) & \rightarrow H^{t+1}\left(V, E^{p}\right) ; \\
\delta^{t, p}: K^{p, t}(V, C) & \approx H^{t}\left(V, E^{p}\right) ; \\
\delta_{S}^{t, p}: K^{p-1, t}(S, C) & \approx H^{t}\left(S, E^{p-1}[S]\right) .
\end{array}
$$

Considérons le diagramme commutatif:

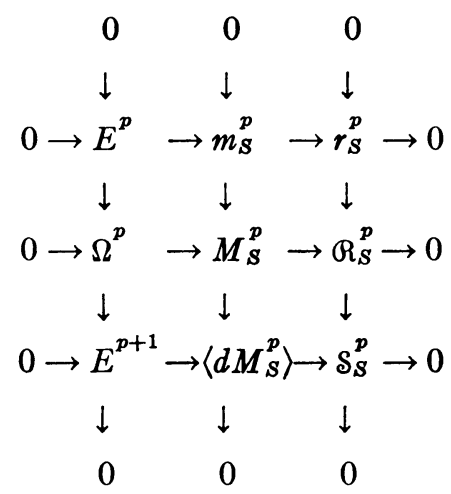

où les suites sont exactes et ont été définies plus haut. (32) définit le diagramme de cohomologie suivant dans lequel les suites sont exactes, le carré $Q$ anticommutatif et les autres carrés commutatifs [1, Chap. IV, Proposition $2.1]$ :

$$
\begin{aligned}
& H^{t+1}\left(V, \Omega^{p}\right) \rightarrow H^{t+1}\left(V, M_{S}^{p}\right) \\
& H^{t}\left(V, s_{S}^{p}\right) \rightarrow H^{t+1}\left(V, E^{p+1}\right) \rightarrow B^{t+1}\left(V,\left\langle d M_{S}^{p}\right\rangle\right) \\
& \begin{array}{l}
\downarrow \quad Q \\
H^{t+1}\left(V, r_{S}^{p}\right) \rightarrow H^{t+2}\left(V, E^{p}\right)
\end{array} \quad \stackrel{\downarrow}{H^{t+2}\left(V, m_{S}^{p}\right)} \\
& \downarrow \\
& H^{t+2}\left(V, M_{S}^{p}\right) \text {. }
\end{aligned}
$$

Si $V$ est kählérienne compacte: $H^{t+1}\left(V, \Omega^{p}\right) \rightarrow H^{t+1}\left(V, E^{p+1}\right)$ a une image nulle d'après la Proposition $1.13 \mathrm{de}[2]$. Si, en outre, $\{S\}$ est suffisamment ample, on a: $H^{t+1}\left(V, M_{S}^{p}\right) \approx H^{t+2}\left(V, M_{S}^{p}\right)=0$ pour $t \geqq 0$, d'après le Théorème 1 de [3]. Alors, du diagramme (33) on déduit le diagramme suivant: 


$$
\begin{aligned}
& H^{t}\left(V, S_{S}^{p}\right) \stackrel{\left.\mu^{\prime}\right) \stackrel{0}{\downarrow}}{\downarrow}{ }^{t+1}\left(V, E^{p+1}\right) \stackrel{0}{\lambda^{\prime}} \stackrel{\downarrow}{\rightarrow} B^{t+1}\left(V,\left\langle d M_{S}^{p}\right\rangle\right) \\
& \downarrow \quad \gamma^{\prime} \downarrow \quad \gamma \downarrow \\
& H^{t+1}\left(V, r_{S}^{p}\right) \stackrel{\mu^{t+1, p}}{\longrightarrow} B^{t+2}\left(V, E^{p}\right) \stackrel{\lambda}{\rightarrow} B^{t+2}\left(V, m_{S}^{p}\right) \\
& \stackrel{\downarrow}{0}
\end{aligned}
$$

dans lequel les suites sont exactes. $k_{3}^{p, t+1}$ étant le conoyau de $\mu^{t t+1, p}$, il est isomorphe à l'image de $\lambda^{\prime}$; l'homomorphisme $\gamma$ étant un isomorphisme, l'image de $\lambda^{\prime}$ est isomorphe à l'image de $\lambda \circ \gamma^{\prime}$.

Du diagramme (34) se déduit le diagramme

$$
\begin{gathered}
0 \\
\downarrow \\
K^{p+1, t+1}(V, C) \\
\bar{\gamma} \downarrow \\
K^{p-1, t+1}(S, C) \stackrel{h^{t+2, p}}{\longrightarrow} K^{p, t+2}(V, C) \stackrel{\bar{\lambda}}{\rightarrow} H^{t+2}\left(V, m_{S}^{p}\right),
\end{gathered}
$$

dans lequel les suites sont exactes et où:

$$
h^{t+2, p}=\left(\delta^{t+2, p}\right)^{-1} \circ \mu^{t+1, p} \circ \nu^{t+1, p} \circ \delta_{S}^{t+1, p} ; \quad \bar{\lambda}=\lambda \circ \delta^{t+2, p}
$$

et

$$
\bar{\gamma}^{\prime}=\left(\delta^{t+2, p}\right)^{-1} \circ \gamma^{\prime} \circ \delta^{t+1, p+1} \text {. }
$$

Alors, $k_{3}^{p, t+1}$ est isomorphe à l'image de $\bar{\lambda} \circ \bar{\gamma}^{\prime}$, c'est-à-dire, à l'image de la restriction de $\bar{\lambda}$, aux classes de premier degré au moins égal à $p+1$; de plus, l'homomorphisme $h^{t+2, p}$ est de type (1.1), donc l'image de $\bar{\lambda} \circ \bar{\gamma}^{\prime}$ et l'espace $k_{3}^{p, t+1}$ sont isomorphes au conoyau de

$$
h^{t+1, p+1}: K^{p, t}(S, C) \rightarrow K^{p+1, t+1}(V, C) \text { si } p>0
$$

et à $K^{1 / t+1}(V, C)$ si $p=0$.

Il en résulte:

$$
\operatorname{dim} k_{3}^{p, t+1}=\operatorname{dim} \text { conoyau } h^{t+1, p+1}=\operatorname{dim} k_{2}^{p+1, t+1},
$$

c'est-à-dire, d'après la Proposition 15:

si $p+1+t<m-2$ ou $p+1+t \geqq m+1$, on a:

$$
\operatorname{dim} k_{3}^{p, t+1}=\operatorname{dim} K^{p+1, t+1}(V, C)-\operatorname{dim} K^{p, t}(S, C) .
$$

si $p+1+t=m$, on a: $\operatorname{dim} k_{3}^{p, m-p}=0$, c.q.f.d. 
8. Conclusions.

THÉORÈME 17. Si $V$ est une variété kählérienne compacte, $S$ un sousensemble analytique principal de $V$ sans singularité, et si $\{S\}$ est suffisamment ample:

(1) Pour $q>0$, on $a: H^{q}\left(V, M_{S}^{p}\right)=0$ et si, en outre:

(a) $p+q<m-3$ ou $p+q \geqq m+1$, on $a$ :

$\operatorname{dim} H^{q}\left(S, \Omega^{p}\left(\{S\}_{S}\right)\right)=\operatorname{dim} H^{p, q+1}(V, C)-\operatorname{dim} H^{p-1, q}(S, C) ;$

(b) $p+q=m$, on $a: \operatorname{dim} H^{m-p}\left(S, \Omega^{p}\left(\{S\}_{S}\right)\right)=0$;

pour $q=0$ et $p<m-3$ ou $p=m$, on $a$ :

$$
\begin{aligned}
\operatorname{dim} H^{0}\left(V, M_{S}^{p}\right)= & \operatorname{dim} H^{p-1,0}(S, C)+\operatorname{dim} H^{0}\left(S, \Omega^{p}\left(\{S\}_{s}\right)\right)-\operatorname{dim} H^{p, 1}(V, C) \\
& +\operatorname{dim} H^{p, 0}(V, C) .
\end{aligned}
$$

(2) Pour $q \geqq 0$ et $p+q<m-2$ ou $p+q \geqq m+2$, on $a$ :

$$
\operatorname{dim} H^{q}\left(V, m_{S}^{p}\right)=\operatorname{dim} K^{p, q}(V, C)-\operatorname{dim} K^{p-1, q-1}(S, C) ;
$$

pour $p+q=m+1: \operatorname{dim} H^{m-p+1}\left(V, m_{S}^{p}\right)=0$.

(3) Pour $p<m-3$, on $a$ :

$$
\operatorname{dim} H^{0}\left(V, d M_{S}^{p}\right)=\operatorname{dim} H^{0}\left(S, \Omega^{p}\left(\{S\}_{S}\right)\right)+\operatorname{dim} K^{p+1,0}(V, C) ;
$$

pour $q>0$ et $p+q<m-3$ ou $p+q \geqq m+1$, on $a$ :

$$
\operatorname{dim} H^{q}\left(V, d M_{S}^{p}\right)=\operatorname{dim} K^{p, q+1}(V, C)-\operatorname{dim} K^{p-1, q}(S, C) ;
$$

pour $p+q=m: \operatorname{dim} H^{m-p}\left(V, d M_{S}^{p}\right)=0$.

Démonstration. Les suites exactes (27), (28), (29) entraînent respectivement:

$\operatorname{dim} H^{q}\left(V, M_{S}^{p}\right)=\operatorname{dim} H^{q}\left(V, \Re_{S}^{p}\right)-\operatorname{dim} H^{q+1}\left(V, \Omega^{p}\right)+\operatorname{dim} k_{1}^{p, q}+\operatorname{dim} k_{1}^{p, q+1} ;$ $\operatorname{dim} H^{q}\left(V, m_{S}^{p}\right)=\operatorname{dim} H^{q}\left(V, r_{S}^{p}\right)-\operatorname{dim} H^{q+1}\left(V, E^{p}\right)+\operatorname{dim} k_{2}^{p, q}+\operatorname{dim} k_{2}^{p, q+1} ;$ $\operatorname{dim} H^{q}(V, d M)_{S}^{p}=\operatorname{dim} H^{q}\left(V, S_{S}^{p}\right)-\operatorname{dim} H^{q+1}\left(V, E^{p+1}\right)+\operatorname{dim} k_{3}^{p, q}$

$$
+\operatorname{dim} k_{3}^{p, q+1} \text {. }
$$

Le théorème résulte alors des isomorphismes des Théorèmes 1.4 et 1.6 de [2], des isomorphismes évidents $k_{1}^{p, 0} \approx H^{0}\left(V, \Omega^{p}\right) \approx H^{p, 0}(V, C) ; k_{2}^{p, 0}$ $\approx H^{0}\left(V, E^{p}\right) \approx K^{p, 0}(V, C) ; k_{3}^{p, 0} \approx H^{0}\left(V, E^{p+1}\right) \approx K^{p+1,0}(V, C)$, et, pour la Partie (1), du Théorème 1 de [3] et des Propositions 7 et 13, pour la Partie (2), des Propositions 4 et 15, pour la Partie (3) des Propositions 8 et 16, de 
l'expression de $\operatorname{dim} H^{q}\left(S, \Omega^{p}\left(\{S\}_{S}\right)\right)$ obtenue au (1) et du fait que, sur les variétés kählériennes compactes $V$ et $S$, on a: $K^{p, q+1}(V, C) \approx H^{p, q+1}(V, C)$ $\oplus K^{p+1, q}(V, C)$ et $K^{p-1, q}(S, C) \approx H^{p-1, q}(S, C) \oplus K^{p, q-1}(S, C)$ [2, Théorème $1.14]$.

THÉOR̀̀me 18. Si $V$ est une variété kählérienne compacte, $S$ un sousensemble analytique principal de $V$ sans singularité, si $\{S\}$ est suffisamment ample et si $p+q<m-3$ ou $p+q \geqq m+1$, on $a$ :

$$
\operatorname{dim} \mathcal{E}_{S}^{p+1, q}=\operatorname{dim} K^{p, q+1}(V, C)-\operatorname{dim} K^{p-1, q}(S, C) .
$$

En particulier, la dimension de l'espace vectoriel des classes de $(p+1)$-formes méromorphes localement exactes dont l'ensemble polaire est $S$ a la multiplicité deux au plus, modulo les différentielles de formes méromorphes sur $V$ dont l'ensemble polaire est $S$ a la multiplicité un au plus est, pour $p<m-3$ :

$$
\operatorname{dim} \mathcal{E}_{S}^{p+1,0}=\operatorname{dim} K^{p, 1}(V, C)-\operatorname{dim} H^{p-1,0}(S, C) .
$$

Démonstration. Si $g>0$, on a: $H^{q}\left(V, M_{S}^{p}\right)=0$, alors, par définition $\mathcal{E}_{S}^{p+1, q} \approx H^{q}\left(V, d M_{S}^{p}\right)$, donc, d'après (3) du Théorème 17 , on a:

$$
\operatorname{dim} \mathcal{E}_{S}^{p+1, q}=\operatorname{dim} K^{p, q+1}(V, C)-\operatorname{dim} K^{p-1, q}(S, C) .
$$

D'après la formule $\left(26^{\prime}\right)$ pour $q=0$, on a:

$$
\operatorname{dim} \mathcal{E}_{S}^{p+1,0}=\operatorname{dim} H^{0}\left(V, m_{S}^{p}\right)-\operatorname{dim} H^{0}\left(V, M_{S}^{p}\right)+\operatorname{dim} H^{0}\left(V, d M_{S}^{p}\right),
$$

donc, pour $p<m-3$, d'après le Théorème 17 et le fait que $K^{p, 1}(V, C)$ $\approx H^{p, 1}(V, C) \oplus K^{p+1,0}(V, C)$ sur la variété kählérienne compacte $V[2$, Théorème 1.14]:

$$
\operatorname{dim} \varepsilon_{S}^{p+1,0}=\operatorname{dim} K^{p, 1}(V, C)-\operatorname{dim} H^{p-1,0}(S, C),
$$

ce qui, compte tenu de la Proposition 12, établit la fin du Théorème 18.

\section{BIBLIOGRAPHIE} 19.

1. H. Cartan and S. Eilenberg, Homological algebra, Princeton Mathematical Series, no.

2. P. Dolbeault, Formes differentielles et cohomologie sur une varieté analytique complexe, Ann. of Math. (à paraitre).

3. K. Kodaira, On a differential-geometric method in the theory of analytic stacks, Proc. Nat. Acad. Sci. U.S.A. vol. 39 (1953) pp. 1268-1273.

4. K. Kodaira and D. C. Spencer, On a theorem of Lefschetz and the Lemma of EnriquesSeveri-Zariski, Proc. Nat. Acad. Sci. U.S.A. vol. 39 (1953) pp. 1273-1278.

5. L. Schwartz, Courant associé d une forme différentielle méromorphe sur une varieté analytique complexe. Colloque de Géométrie différentielle, Strasbourg, 1953, pp. 185-195

6. D. C. Spencer, Cohomology and the Riemann-Roch theorem, Proc. Nat. Acad. Sci. U.S.A. vol. 39 (1953) pp. 660-669.

Paris, France. 